\title{
Transient Tidal Circulation and Momentum Balances at a Shallow Inlet
}

\author{
James L. Hench AND Richard A. Luettich JR. \\ Institute of Marine Sciences, University of North Carolina at Chapel Hill, Morehead City, North Carolina
}

(Manuscript received 18 June 2002, in final form 23 October 2002)

\begin{abstract}
An analysis of transient momentum balances is carried out to elucidate circulation, dynamics, and exchange mechanisms at shallow barotropic tidal inlets. Circulation is computed using a depth-integrated, fully nonlinear, time-stepping, finite-element model with variably spaced grids having horizontal resolution down to $50 \mathrm{~m}$. Velocity and elevation fields from the model are used to directly evaluate the contribution of each term in the momentum equations to the overall momentum balance. A transformation of the $x-y$ momentum terms into an $s-n$ coordinate system is used to simplify the interpretation of the dynamics and provide vivid illustrations of the forces and resulting accelerations in the flow. The analysis is conducted for an idealized inlet and contrasted with a highly detailed model of Beaufort Inlet, North Carolina. Results show that momentum balances in the immediate vicinity of these inlets vary significantly in time and space and oscillate between two dynamical states. Near maximum ebb or flood, the alongstream momentum balances are dominated by advective acceleration, pressure gradient, and bottom friction. Cross-stream balances are dominated by centrifugal acceleration and pressure gradients. Near slack, balances more closely follow linear wave dynamics, with local accelerations balancing pressure gradients, and (to a lesser degree) Coriolis. Comparisons between the idealized inlet and Beaufort Inlet show broad similarities in these momentum balances. However, natural inlet geometry and bottom topography, as well as the tidal transmission characteristics of the sounds behind Beaufort Inlet produce strong asymmetries. Moreover, momentum balances are highly localized, often with subkilometer length scales. The dynamics are used to explain the physical mechanisms for inlet exchange. In particular, the results indicate that the cross-stream dynamics generate a "wall" along the length of an inlet during the stronger phases of the tide. The wall is established by opposing cross-inlet pressure gradients and centrifugal forces, and it poses a significant barrier to cross-inlet exchange during the stronger phases of the tide but is absent near slack.
\end{abstract}

\section{Introduction}

Tidal inlets are important conduits between estuarine waters and the coastal ocean for the movement of contaminants and nutrients (e.g., Signell and Butman 1992; Sheng et al. 1996), sediments (e.g., Fenster and Dolan 1996), and biota (e.g., Weinstein 1988; Crowder and Werner 1999). Inlets are common coastal features; one recent study indicates there are $>150$ inlets along the United States coast alone (Carr and Kraus 2002). Their total number worldwide is unknown, but they are found on all continents and particularly along barrier islandsound systems, which compose about $13 \%$ of the world coastline (Cromwell 1973). Many reef passes also possess inlet features (e.g., Wolanski et al. 1988). Since inlets are the primary locales for exchange between sounds (also called lagoons or bays) and the coastal ocean they are naturally of great interest. However, an understanding of exchange processes is based implicitly on detailed knowledge of the circulation (Geyer and

Corresponding author address: James L. Hench, Institute of Marine Sciences, University of North Carolina at Chapel Hill, 3431 Arendell St., Morehead City, NC 28557.

E-mail: hench@unc.edu
Signell 1992). A complete dynamical description of inlet circulation has proven elusive as the circulation fields are spatially complex, transient, and nonlinear.

In the earliest work on inlet exchange and kinematics, Stommel and Farmer (1952) noted the distinct difference between flood (resembling a potential sink) and ebb (resembling a jet) circulation outside an inlet. These basic flow patterns have subsequently been reproduced in numerical models of idealized inlets (e.g., Kapolnai et al. 1996; Wheless and Valle-Levinson 1996). Circulation models of natural inlets have also been developed (Blanton et al. 1999; Luettich et al. 1999; Brown et al. 2000) and have shown that irregular bathymetry and shoreline geometry can produce highly asymmetric circulation patterns that differ substantially from idealized inlets. Direct observations of circulation in natural inlets also indicate that the spatial and temporal flow structures are more complicated than in idealized inlets (e.g., Kjerfve and Proehl 1979; Takasugi et al. 1990; Chadwick and Largier 1999a,b; Churchill et al. 1999). Baroclinic effects have also been explored, at least for idealized inlets (Chao 1990; Kapolnai et al. 1996; Wheless and Valle-Levinson 1996).

Concurrent with the work on inlet circulation and 
kinematics, progress has been made in describing the dynamics of inlet flow. Imasato (1983) used results from a two-dimensional barotropic idealized inlet model to construct a simple set of conceptual force balance cartoons. Imasato (1987) used the same model to compute selected momentum terms along a single transect adjacent to an inlet. These ideas were extended by Imasato et al. (1994) who used a three-dimensional idealized inlet model to compute vertical profiles of selected momentum terms at two points within the inlet. The most complete dynamical analysis to date appears to be by Ridderinkhof (1988), who computed term-by-term momentum balances for a two-dimensional model of Wadden Sea inlets. However, his analysis was confined to the limiting cases of steady flow (near maximum ebb and maximum flood). These studies have provided much insight into inlet dynamics, although they were all limited in space, time, and/or the portion of the dynamics analyzed.

In this paper, we extend these prior studies with a detailed and systematic examination of the transient momentum balances at two complementary shallow tidal inlets. Model results from both inlets are dissected to assess the contribution of each term in the momentum equations to gain an understanding of the space-time patterns of the dynamics. An idealized inlet is used to identify generic behaviors. These results are contrasted with those from a highly detailed model of a natural inlet to illuminate the confounding roles of irregular bottom topography and shoreline geometry. Herein, we 1) describe the numerical models and the streamline coordinate system used for the momentum balance analysis, 2) describe the modeled circulation fields, 3) analyze the transient momentum balances over a partial tidal cycle, and 4) discuss the dynamics and their implications for inlet exchange.

\section{Methods}

\section{a. Numerical model formulation}

As a necessary step toward understanding time-dependent, three-dimensional, baroclinic momentum balances on irregular bathymetry and geometry, we focus here on barotropic dynamics and assume density gradient effects are dynamically small. These conditions are common at shallow inlets where vertical mixing is strong, and is often the case at Beaufort Inlet, North Carolina, which is the shallow inlet prototype considered in this study. Assuming barotropic conditions and small vertical shears, we solve the fully nonlinear, shallow-water equations using the circulation model ADCIRC (Luettich et al. 1992). In the absence of wind and tidal potential forcing, and assuming a constant lateral viscosity, the governing continuity and momentum equations used in the model are

$$
\frac{\partial \eta}{\partial t}+\frac{\partial U H}{\partial x}+\frac{\partial V H}{\partial y}=0
$$

$$
\begin{aligned}
& \frac{\partial U}{\partial t}+U \frac{\partial U}{\partial x}+V \frac{\partial U}{\partial y}-f V+g \frac{\partial \eta}{\partial x}-\nu \nabla^{2} U \\
& +\left(\frac{C_{f} \sqrt{U^{2}+V^{2}}}{H}\right) U=0 \\
& \frac{\partial V}{\partial t}+U \frac{\partial V}{\partial x}+V \frac{\partial V}{\partial y}+f U+g \frac{\partial \eta}{\partial y}-\nu \nabla^{2} V \\
& +\left(\frac{C_{f} \sqrt{U^{2}+V^{2}}}{H}\right) V=0,
\end{aligned}
$$

where $x$ and $y$ are horizontal coordinates aligned east and north directions, respectively; $U(x, y, t), V(x, y, t)$ are depth-integrated velocities; $H(x, y, t)=h(x, y)+$ $\eta(x, y, t)$ is the total water column height; $\eta(x, y, t)$ is the vertical displacement of the surface from still water; $f$ is the Coriolis parameter; $g$ is the gravitational constant; $\nu$ is the lateral eddy exchange coefficient; $\nabla^{2}=$ $\partial^{2} / \partial x^{2}+\partial^{2} / \partial y^{2}$ is the horizontal diffusion operator; and $C_{f}$ is the quadratic bottom friction coefficient. Equation (1) is transformed into a wave-equation formulation, and the resulting coupled system of equations is discretized using a finite-element method in space and a finite-difference scheme in time (see Luettich et al. 1992). The model has been previously verified in studies of natural tidal inlets (Luettich et al. 1999; Militello and Zarillo 2000) and should faithfully simulate the physics of barotropic flow.

\section{b. Numerical model domains}

Two inlet models with differing degrees of geometric and bathymetric complexity were used. The first is an idealized inlet model, which was constructed to correspond to the general features of Beaufort Inlet, with a domain comprised of two basins connected by an inlet that is $1 \mathrm{~km}$ wide and $0.5 \mathrm{~km}$ long (Fig. 1). Water depths in the sound and inlet were set uniformly to $5 \mathrm{~m}$. Offshore the depth increased linearly from 5 to $14 \mathrm{~m}$ at the open ocean boundary. Flow separation and adverse pressure gradients are significant flow features in inlet problems and adequate grid resolution is essential for accurately modeling these processes. The finite-element method and the use of unstructured grids are particularly useful for studying inlet circulation since they permit selective resolution of a wide range of length scales and complex shoreline geometries while keeping computational requirements tractable. For the idealized inlet model, horizontal grid resolution varied from $1 \mathrm{~km}$ at the open ocean boundary to a uniform $50 \mathrm{~m}$ in the vicinity of the inlet.

The second model is of Beaufort Inlet, which has an inlet width of about $1 \mathrm{~km}$ at its narrowest point and a nominal length of $0.5 \mathrm{~km}$ (Fig. 2). Depths range from 2 to $10 \mathrm{~m}$ along the ebb delta, while the inlet's main channel (dredged for navigation) is about $15 \mathrm{~m}$ at the deepest. The flood delta is cut by several connecting sloughs. Model bathymetry came from a 1998 National 

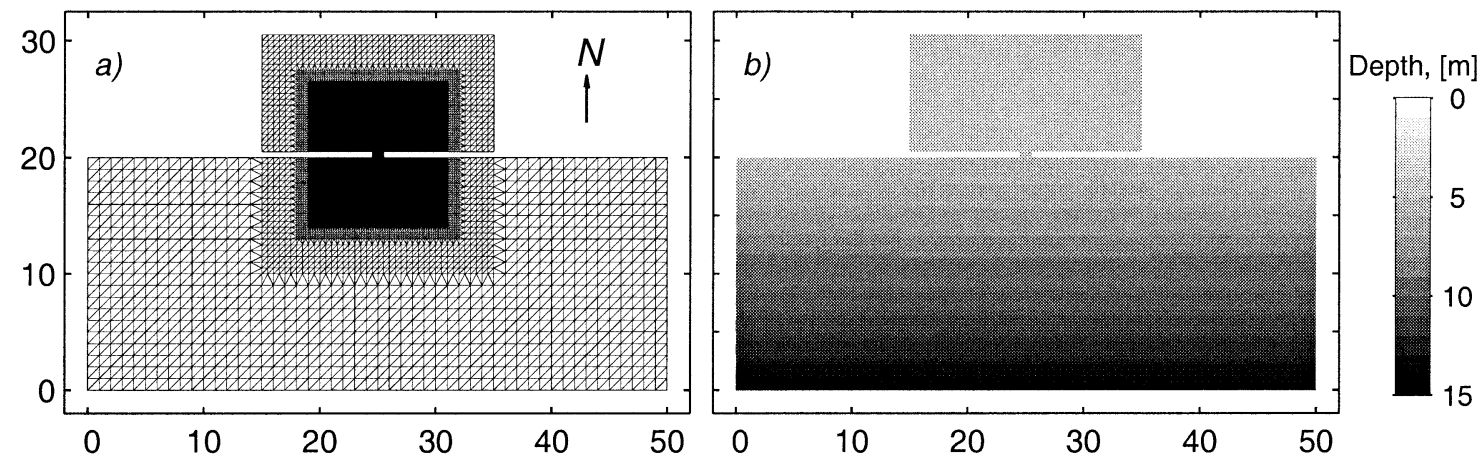

FIG. 1. Idealized inlet (a) model finite-element grid and (b) bathymetry. The grid contains 48273 nodes and 95884 elements. Axes distances are in kilometers.

Oceanic and Atmospheric Administration (NOAA) survey (at $85-\mathrm{m}$ track spacing) and was supplemented in near inlet areas with bathymetry collected with our own research vessel (at 50-m track spacing). Near inlet shorelines were specified with global positioning satellite (GPS) surveys conducted in 1998, consistent with the bathymetric data. Nodal spacing ranged from about 2 $\mathrm{km}$ offshore to $<25 \mathrm{~m}$ in the sound, while grid resolution in the inlet was nominally $50 \mathrm{~m}$.
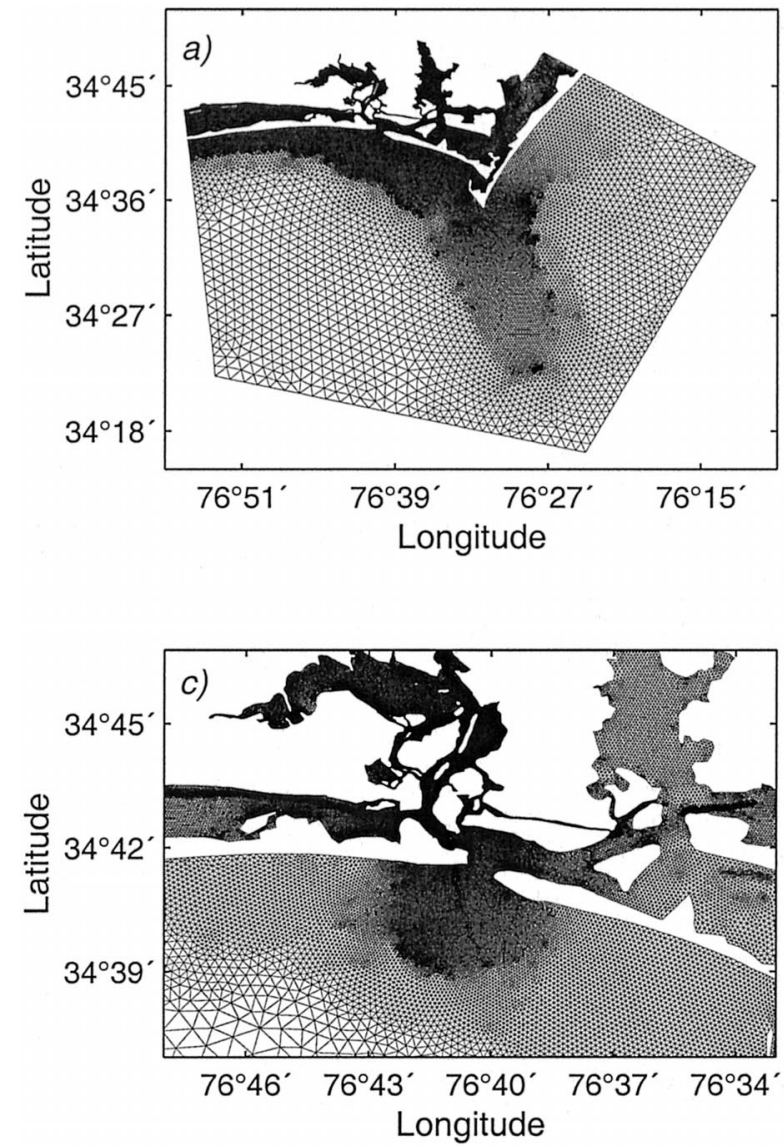

\section{c. Numerical model forcing, run parameters, and verification}

The idealized inlet was forced at the offshore ocean boundary with specified elevations of $0.15-\mathrm{m}$ amplitude and zero phase at the $M_{2}$ frequency. This forcing was selected to produce maximum velocities in the inlet throat of about $1 \mathrm{~m} \mathrm{~s}^{-1}$ (comparable to Beaufort Inlet). Zero normal-flow boundary conditions were imposed

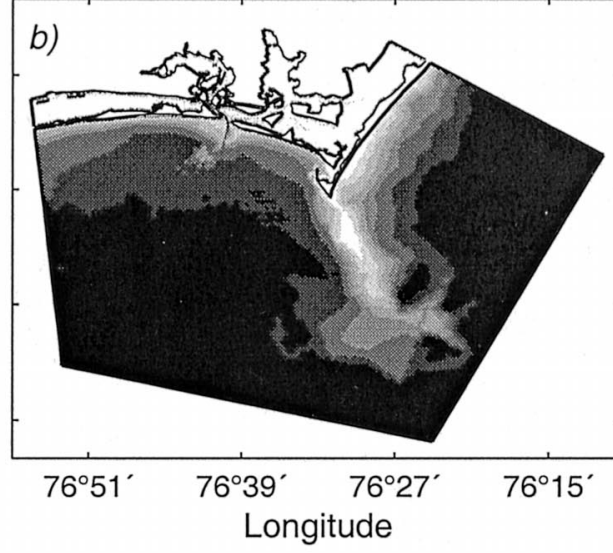

Depth, [m]
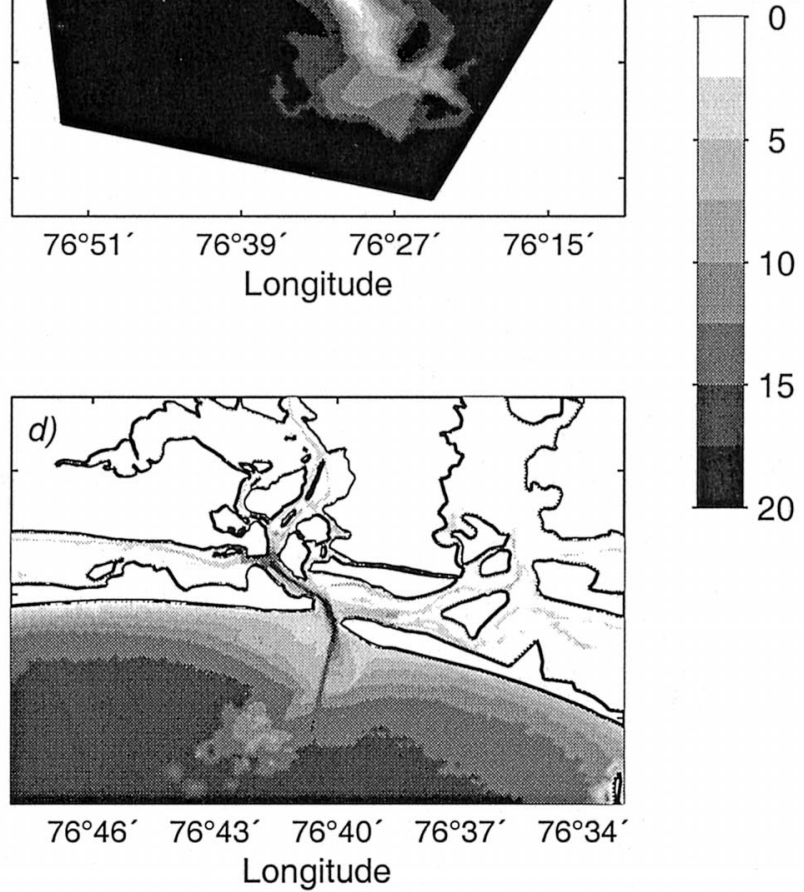

FIG. 2. Beaufort Inlet (a) model finite-element grid and (b) bathymetry. (c), (d) Zoom of inlet shows high-grid-resolution areas. The grid contains 53505 nodes and 102228 elements. 
on land boundaries, the sound, and along the shoreperpendicular offshore boundaries to obtain onshoreoffshore tidal propagation as seen at Beaufort Inlet, and elsewhere along the South Atlantic Bight (Redfield 1958; Pietrafesa et al. 1985). The Beaufort Inlet model was forced at three open boundaries (two within the sound and one on the ocean; see Fig. 2) with specified elevations for the $M_{2}, M_{4}, M_{6}$, and steady tidal constituents obtained from the larger domain circulation model of Luettich et al. (1999). Zero normal-flow boundary conditions were imposed along land boundaries. Both models used constant $C_{f}=0.0025, \nu=7$ $\mathrm{m}^{2} \mathrm{~s}^{-1}$ (the minimum for model stability) and a time step of $2 \mathrm{~s}$. The Coriolis parameter in both models was set to a value corresponding to latitude $34.5^{\circ} \mathrm{N}$. The models were spun up for 6 days to dynamic equilibrium; results from days 6-8 were harmonically analyzed (using $M_{2}, M_{4}, M_{6}, M_{8}, M_{10}$, and steady constituents) to obtain tidal constituents and for the momentum balance analyses.

In our initial modeling efforts at Beaufort Inlet (Luettich et al. 1999), model results compared well with moored instrument data from 16 elevation and 10 velocity stations distributed within the sound. Drogue observations during flood tide (Churchill et al. 1999) also provided encouraging comparisons with the earlier model. The model used in the present study uses a subdomain of the previous model and includes several refinements. High-resolution bathymetry and updated shoreline geometry were incorporated into the grid near Beaufort Inlet (described above), and grid resolution was doubled to better resolve small-scale (subkilometer) flow features.

\section{d. Model sensitivity}

Model runs were conducted to determine sensitivity to parameter values and grid resolution. The Beaufort Inlet model was much more computationally difficult due to the steep bathymetry and geometric complexity and was the focus of our sensitivity studies.

In selecting a lateral viscosity, we followed the philosophy of Geyer and Signell (1992): use as little viscosity as possible (in combination with high grid resolution) in order to model advective processes explicitly. We reran the Beaufort Inlet model with $\nu=10 \mathrm{~m}^{2} \mathrm{~s}^{-1}$ and compared tidal ellipse parameters at about 500 uniformly spaced stations within a $3-\mathrm{km}$ radius of the Inlet. Rms differences for $M_{2}$ semimajor axes (SEMA) were $2 \%$, and $M_{2}$ velocity phases (PHA) differed by $8 \%$. The higher harmonics were more affected with $12 \%$ differences in $M_{4}$ SEMA and $21 \%$ difference for $M_{4}$ PHA.

We used the canonical drag coefficient value 0.0025 because previous model verification work (Luettich et al. 1999) showed this value provided good agreement with observed tidal constituents. A run with $C_{f}=0.0030$ showed 5\% difference in $M_{2}$ SEMA and $10 \%$ difference in $M_{2}$ PHA. Again, the higher harmonics were more affected with $17 \%$ difference in $M_{4}$ SEMA and $21 \%$ in $M_{4}$ PHA. Here, $M_{6}$ changed the most with $16 \%$ difference in SEMA and 55\% in PHA; this was expected since $M_{6}$ is primarily excited by nonlinear bottom friction. However, the change to the overall flow is fairly insignificant since $M_{6}$ makes up $<10 \%$ of the total velocity.

Grid resolution effects were studied by rerunning the Beaufort Inlet model with double the resolution in the near-inlet region $(25 \mathrm{~m})$. Tidal harmonics were interpolated to a common set of about 500 uniformly spaced near-inlet stations, and rms differences between the runs were: $0.5 \% M_{2}$ SEMA and $1 \% M_{2}$ PHA, $2 \% M_{4}$ SEMA and $4 \% M_{4}$ PHA, and $2 \% M_{6}$ SEMA and $9 \% M_{6}$ PHA. The higher harmonics showed greater differences as they benefited more from increased resolution of the shorter wavelengths.

Based on the relative magnitudes of the $M_{2}, M_{4}$, and $M_{6}$ velocities, the total model response in these sensitivity studies was $<10 \%$ of the baseline run. We conclude that the Beaufort Inlet model results were not overly sensitive within a reasonable range of model parameters. The grid is highly converged for the barotropic $M_{2}$ tide, as well as the major nonlinear overtides (and tidal residuals). Sensitivity runs with the idealized inlet showed even smaller differences.

\section{e. Momentum balance calculations}

Velocity and elevation fields from the models were used to reconstruct momentum terms at each computational node throughout a tidal cycle. Each term was evaluated using exactly the same integration, assembly scheme, and run parameters as in the circulation model (see Luettich et al. 1992 for details) so that the individual momentum terms would each be consistent with the computed flow fields. To simplify dynamical interpretation, the $x-y$ components of each term in equations (2) and (3) were rotated at each node into a local streamwise-normal $(s-n)$ coordinate system aligned with the instantaneous velocity vector. This "streamline" coordinate system was defined with the $s$ direction positive in the direction of flow, and the $n$ direction positive left of the flow. The local acceleration terms were treated with a forward-Euler two time-level finite difference scheme, where the streamline velocities and angles were defined relative to the initial time level. The appendix provides a derivation for the $x-y$ to $s-n$ transformation. The $s-n$ momentum equations and their physical interpretations are

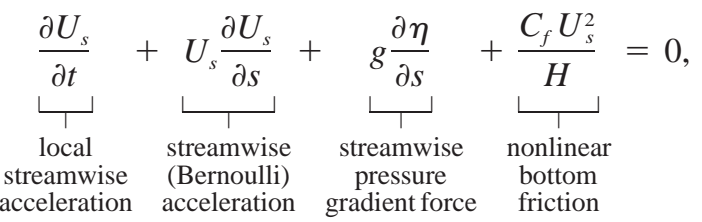




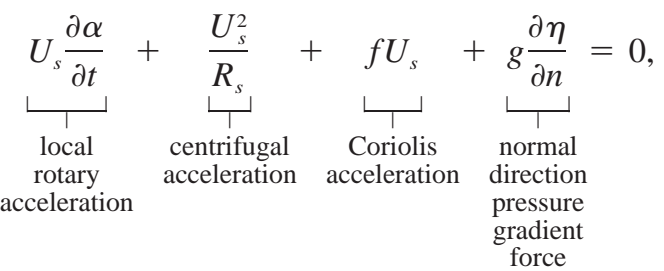

where $U_{s}(x, y, t)$ is the streamwise velocity, $\alpha(x, y, t)$ is the streamline angle (the angle between the positive $x$ axis and the local flow vector), and $R_{s}(x, y, t)$ is the streamwise radius of curvature. With this choice of coordinate system there is, by definition, no normal component to the flow (i.e., $U_{n}=0$ everywhere at all times). Therefore the Coriolis term is zero in the $s$ equation, as is the bottom friction term in the $n$ equation. Moreover, the advective acceleration terms collapse to a single term in each equation: streamwise in the $s$ equation and centrifugal in the $n$ equation. For both the idealized and Beaufort inlet models, momentum was conserved to within one percent before and after the transformation. The horizontal diffusion terms were generally an order of magnitude smaller than the other terms, and for simplicity are omitted from Eqs. (4) and (5) as well as from the discussion below. Results are presented in terms of momentum fluxes (obtained by multiplying each term by $H$ ) to provide a more physically intuitive picture of the momentum balances.

\section{Circulation}

Modeled inlet circulation fields are shown in Fig. 3 in terms of major tidal constituents. For both inlets, $M_{2}$ tidal ellipses are largest in the inlet throat and rapidly diminish within several kilometers from the inlet (Figs. $3 \mathrm{a}, \mathrm{b})$. On both the sound and ocean sides, $M_{2}$ ellipse orientations are directed toward the inlets, and maximum velocities are adjacent to the headland tips rather than at the inlet centers. The $M_{2}$ ellipses are highly rectilinear in the inlet throat and become more rotary with increasing distance from the inlet.

The strong nonlinear nature of inlet flow generates significant overtides and tidal residuals. Lateral shear in an inlet is generated during both ebb and flood so the principal quarter-diurnal overtide, $M_{4}$, should coincide (at least qualitatively) with advective acceleration patterns (Parker 1991). For the idealized inlet $M_{4}$ ellipses are largest adjacent to the headlands features and weakest in the inlet throat, where streamlines become straight (Fig. 3c). At Beaufort Inlet, $M_{4}$ ellipses are also largest near the headland tips, but in contrast to the idealized inlet there are significant regions offshore with large $M_{4}$ (Fig. 3d). Beaufort Inlet model runs with uniform flat bathymetry ( $h=7 \mathrm{~m}$ ) show that natural topography (and thus differential bottom friction) generates a considerable part of the lateral shear and this suggests that topography is the source of enhanced $M_{4}$ relative to the idealized inlet.
The principal sexdiurnal overtide, $M_{6}$, is primarily generated by nonlinear bottom friction (Parker 1991). For the two inlets, $M_{6}$ magnitudes are comparable to $M_{4}$ magnitudes, but the spatial distributions are different. At the idealized inlet $M_{6}$ is largest within the inlet throat, where flow speeds are largest. For Beaufort Inlet, the largest $M_{6}$ is not in the inlet throat, but rather in the shallow sound just east of the inlet, where flow speeds are highest and depths are shallow. The higher harmonics of $M_{8}$ and $M_{10}$ (not shown) show spatial patterns similar to $M_{4}$ and $M_{6}$, respectively, but were at least a factor of 2-10 smaller than both and thus contribute little to the total velocity signal.

Nonlinear flow also generates Eulerian residual currents and both inlets exhibit quadrapole residual fields (Figs. 3g,h). The idealized inlet field is nearly symmetric, while Beaufort Inlet shows a pronounced offshore anticyclonic residual eddy west of the inlet and a rather weak eastside eddy. One might expect this asymmetry to be due to Coriolis enhancing the westside eddy and diminishing the eastside eddy. However, inspection of the idealized inlet shows this is a minimal effect, as did a Beaufort Inlet run with $f=0$. Another possible asymmetry source is phase differences in the offshore open boundary forcing. However, the maximum difference in $M_{2}$ elevation phase along the open boundary is less than $1.6^{\circ}$ (about a 6-min phase lag). Rerunning the model with uniform phases (set to the mean values) yielded results nearly the same as those using the actual forcing (e.g., residual speed and direction changed by $<3 \%$ in the immediate vicinity of the inlet). These results suggest that the asymmetries are principally due to bathymetric and geometric effects. In the throat of Beaufort Inlet there is a net inflow on the east side and outflow on the west side. East and west of the inlet on the sound side are counterrotating eddies, which appear to be significantly constrained by the land boundaries.

\section{Momentum balances}

Momentum balances were computed at each model time step over a complete semidiurnal tidal cycle. Animations of these results revealed that the most salient time-varying features can be seen by examining three phases of the tide: maximum ebb, midebb, and slack before flood. Figure 4 shows the phases of the tide to be discussed for both inlets. For the idealized inlet, velocity and elevation fields at the inlet center are nearly $90^{\circ}$ out of phase, indicative of a standing wave. The corresponding figure for Beaufort Inlet indicates more of a progressive wave, with velocity leading elevation by about $1.5 \mathrm{~h}$. The more progressive nature of the tide at Beaufort Inlet is due to the extensive shallow sound that is less reflective than the sound in the idealized inlet model. In the analysis below, the phase of the tide is defined relative to a point at the geometric center of each inlet. 
M2

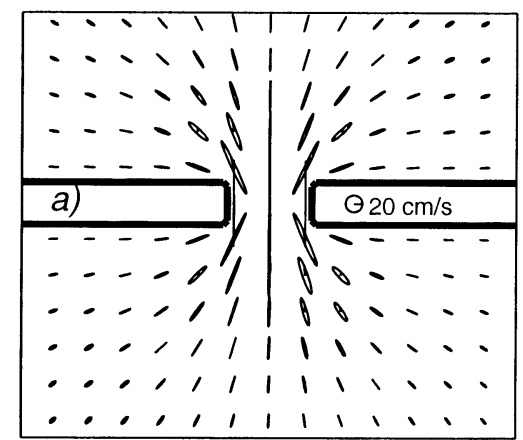

M4

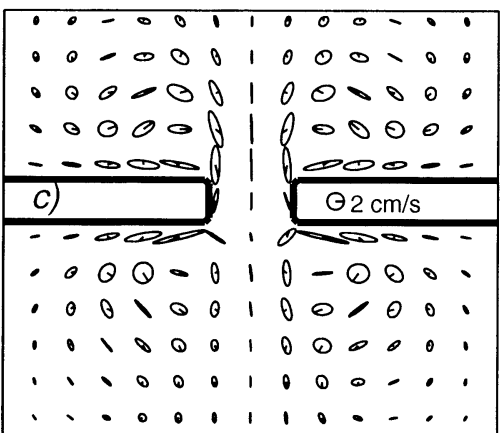

M6

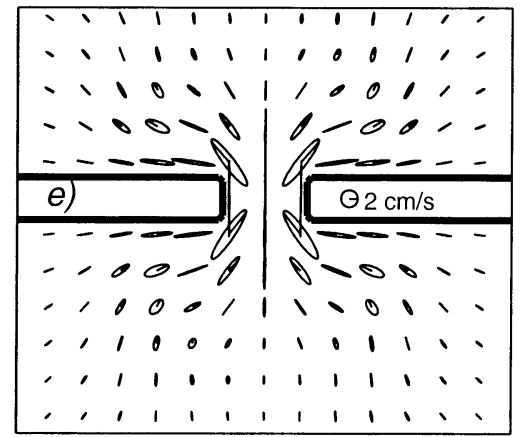

Res.

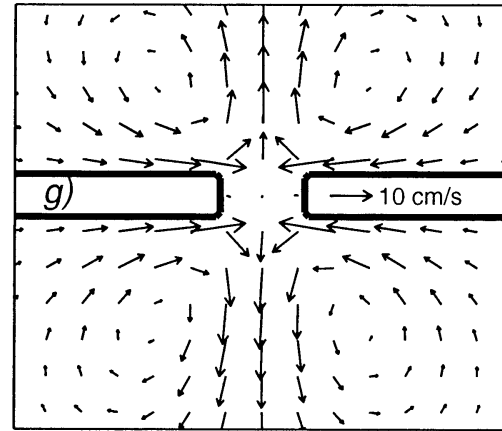

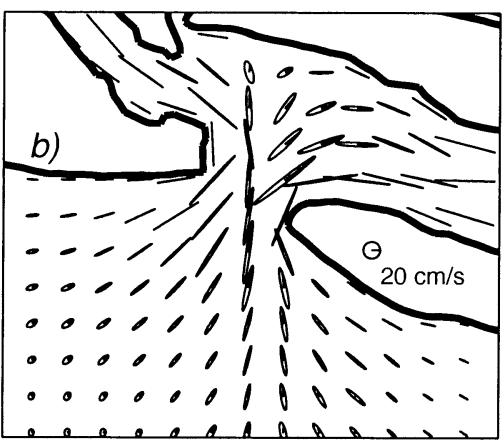
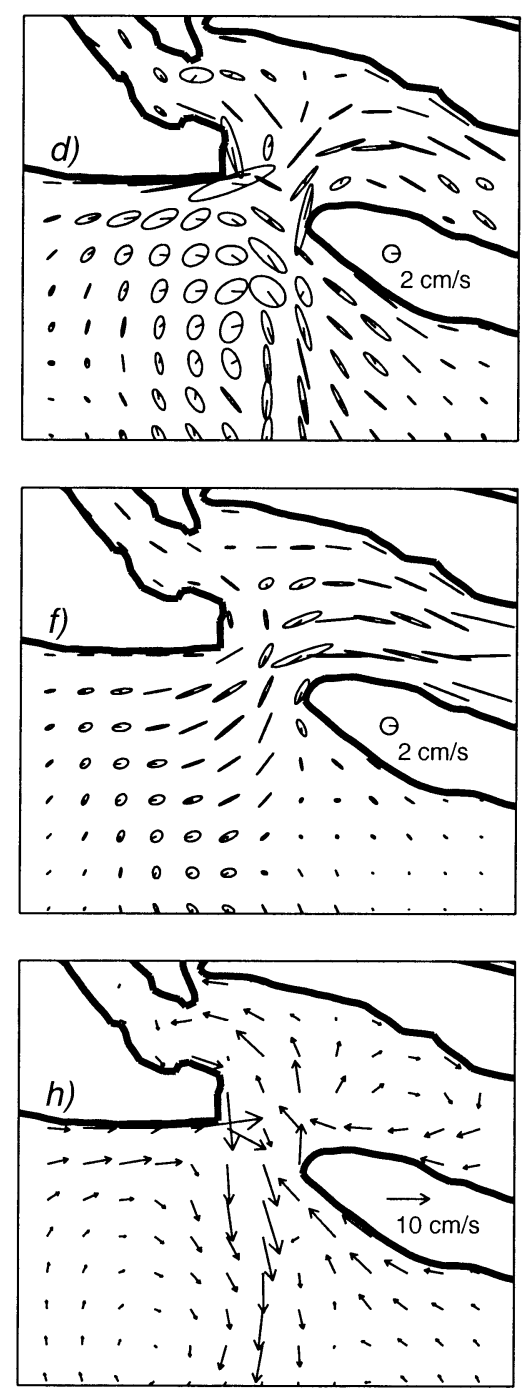

FIG. 3. (a)-(f) Tidal ellipses and (g),(h) Eulerian residual currents for the (left) idealized inlet and (right) Beaufort Inlet in terms of major tidal constituents: (a), (b) $M_{2}$; (c), (d) $M_{4}$; and (e), (f) $M_{6}$. Model results were interpolated onto a coarser uniform grid (with 425-m spacing) for clarity. Note that the $M_{2}$ scale is different than for $M_{4}$ and $M_{6}$.

\section{a. Maximum ebb}

Figure 5 shows the contribution of each term in the $s$ and $n$ direction momentum equations for the idealized inlet at maximum ebb. At this tide phase the flow has reached a point of near steady state, and the local acceleration term (Fig. 5d) is close to zero indicating little change in flow speed, while the rotary acceleration (Fig. 5h) shows little change in flow direction. Streamwise 

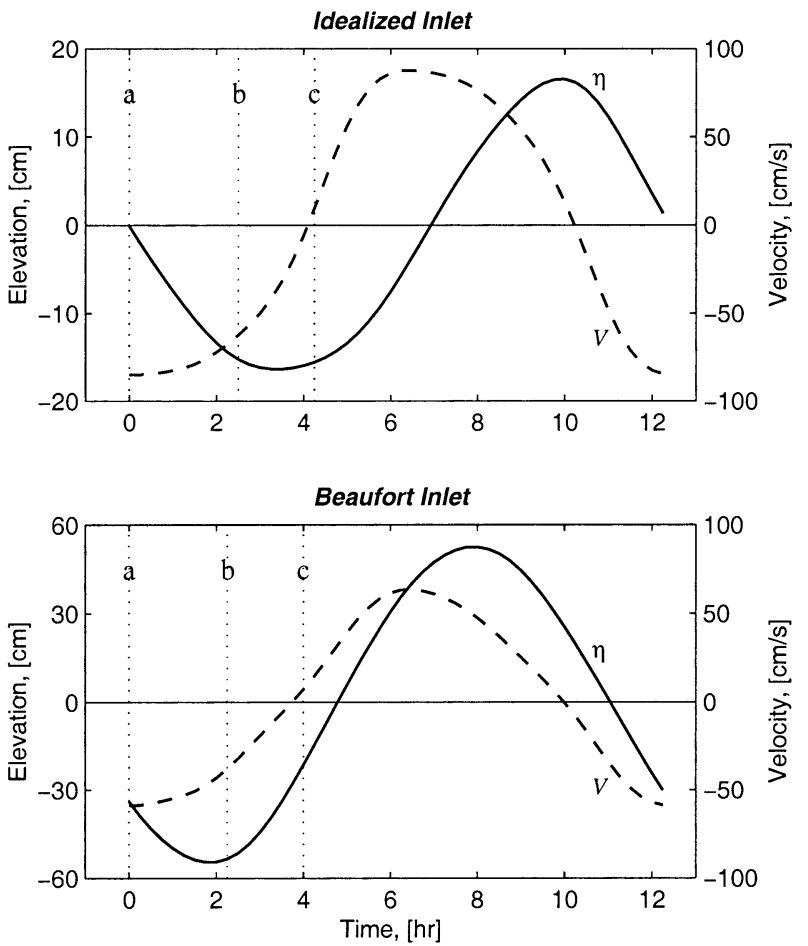

FIG. 4. Inlet elevation $(\eta)$ and velocity $(V)$ time series at the inlet centers for one tidal cycle: (top) idealized; (bottom) Beaufort. Times selected for detailed momentum balance analysis are indicated with vertical lines: (a) maximum ebb, (b) midebb (1.50 h before slack), and (c) slack before flood ( $0.25 \mathrm{~h}$ after slack).

advective acceleration (Fig. 5e) is large and positive as the flow enters the inlet from the sound, and large and negative as the flow exits. A large negative (i.e., favorable) streamwise pressure gradient drives the flow from the sound toward the inlet (Fig. 5f). (There is about $6-\mathrm{cm}$ elevation difference between the sound and ocean.) The water surface in the inlet dips as the flow accelerates through the inlet (see Fig. 5b), but the flow remains subcritical (maximum Froude numbers $<0.8$ ). This Bernoulli-type effect (i.e., streamwise advectionpressure gradient balance) yields an adverse pressure gradient as the flow exits the inlet (Fig. 5f). Outside the inlet throat transition area are two distinct dynamical zones. In the ebb jet, bottom friction balances the streamwise advective acceleration (Figs. 5e,g), while in the sink region on the sound side streamwise pressure gradient balances streamwise advective acceleration (Figs. 5e,f).

In the direction normal to the flow, the primary balance is between centrifugal acceleration (Fig. 5i) and the cross-stream pressure gradient (Fig. 5j). On the east side of the inlet, centrifugal acceleration is positive, reflecting cyclonic turning of the flow; the converse is true on the west side. The cross-stream pressure gradient is positive on the inlet's west side (indicating an upward slope eastward) and negative on the inlet's east side (indicating a downward slope eastward). Thus there is a dome of water across the inlet where the water surface has adjusted to opposing centrifugal accelerations. Model results indicate that the water level is about $3 \mathrm{~cm}$ higher at the inlet center than at the sides (see elevation contours in Fig. 5b). The centrifugal acceleration and cross-stream pressure gradient balance relaxes within about $1 \mathrm{~km}$ from the inlet. Coriolis (Fig. 5k) is strongest (in the absolute sense) in the inlet throat, where streamwise velocities are largest, but is relatively weak compared to the centrifugal acceleration and normal direction pressure gradient. Further away from the inlet Coriolis increases in relative importance (see section $4 \mathrm{~d}$ below).

Beaufort Inlet maximum ebb momentum fluxes are shown in Fig. 6. As with the idealized inlet, flow is near steady state and local and rotary accelerations (Figs. $6 \mathrm{~d}, \mathrm{~h})$ are close to zero in the vicinity of the inlet. The exception is in the offshore portion of the deep navigation channel where flow speeds are still increasing. Near-inlet streamwise advective accelerations (Fig. 6e) are quite strong; positive as the flow from the sound enters the inlet and negative as the flow exits the inlet. In contrast to the idealized inlet, there are marked localized areas of negative advective acceleration at the tips of headland features associated with flow separation, which are balanced by local adverse pressure gradients and bottom friction (Figs. 6e,f,g). These local dynamics are similar to those found around the single headland of Signell and Geyer (1991). Comparable to the idealized inlet there is a $6-\mathrm{cm}$ elevation difference between the sound and ocean, and this streamwise pressure gradient drives the ebb. Outside the inlet is a region of large streamwise deceleration and positive (i.e., adverse) pressure gradient arising from a Bernoulli effect. Unlike the idealized inlet, this region is displaced south of the geometric center of the inlet. On the sound side, bottom friction is greatest in the deep navigation channel west of the inlet and in the narrow slough east of the inlet (Fig. 6g). This term rapidly diminishes in importance away from the inlet, where the dynamics more closely follow linear wave theory.

In the cross-stream direction, centrifugal acceleration (Fig. 6i) is largest where flow from the sound turns toward the inlet throat and follows a pattern remarkably similar to the idealized inlet. Offshore of the inlet the navigation channel turns slightly westward, so flow exiting the inlet's east side veers toward the west, and the centrifugal acceleration changes sign to an anticyclonic orientation. As with the idealized inlet, the centrifugal acceleration-pressure gradient balance yields a dome of high water across the inlet throat. However unlike the idealized inlet, the dome is highly asymmetric, with a 3 -cm elevation difference from the west side to the center, and an $8-\mathrm{cm}$ difference from the east side to the center (Fig. 6b). The asymmetry is due to stronger centrifugal acceleration on the east side where $R_{s}$ is much smaller. Coriolis (Fig. 6k) plays a secondary role to the centrifugal acceleration and cross-stream pressure gra- 


\section{Idealized Inlet}
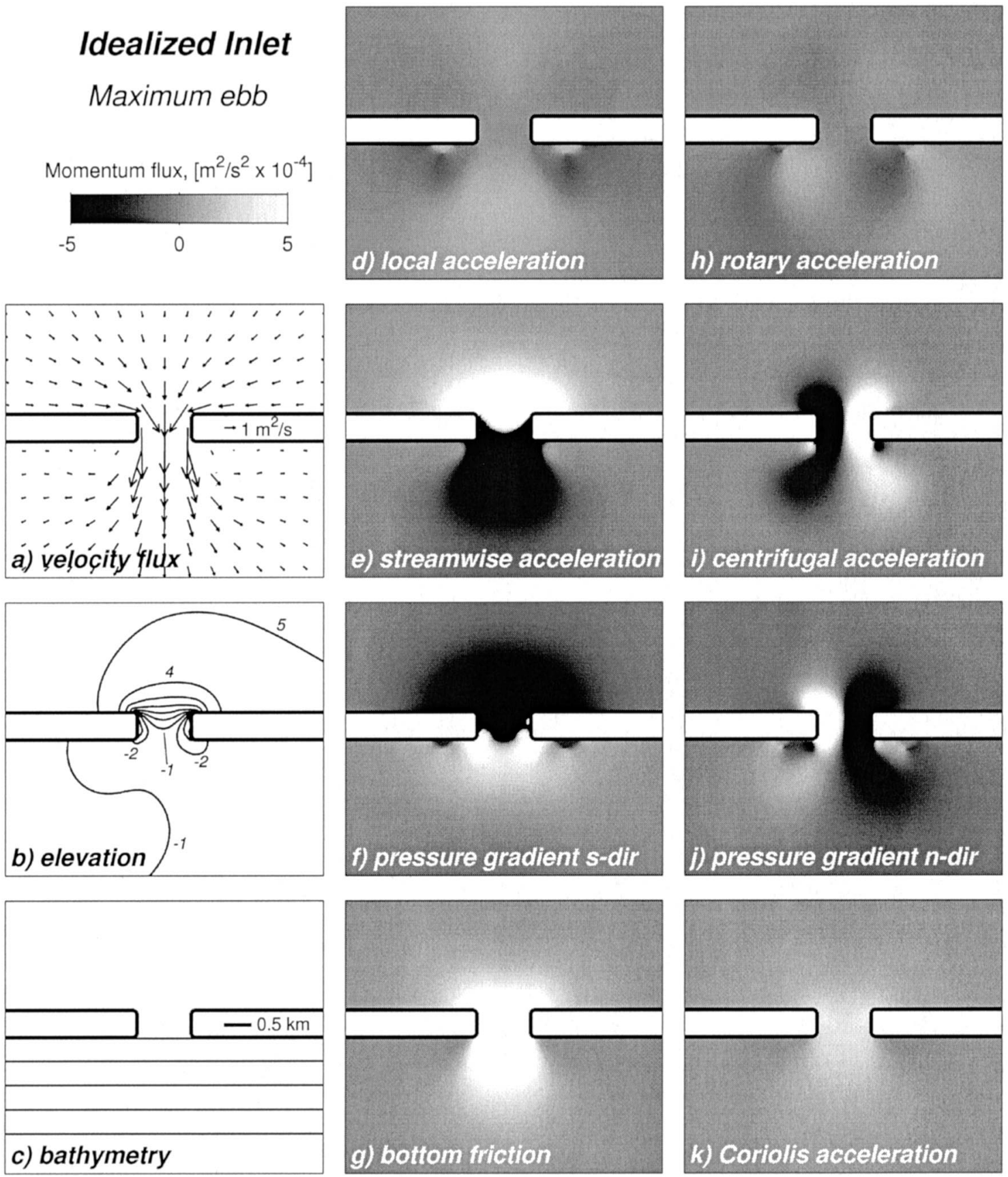

FIG. 5. Circulation and momentum balances for idealized inlet at maximum ebb: (a) velocity flux (depth-averaged velocity multiplied by total water column) vectors interpolated onto uniform 425-m grid for clarity, (b) free-surface elevation with 1$\mathrm{cm}$ contour intervals, (c) bathymetry with $1-\mathrm{m}$ contour intervals, and (d)-(k) shaded contours of individual momentum flux terms (see text for description).

dient at maximum ebb, but is largest in the navigation channel where flow speeds are greatest.

\section{b. Midebb}

As ebb advances toward slack, the pressure gradient between the sound and ocean weakens. For the idealized inlet the elevation difference between sound and ocean is about $2 \mathrm{~cm}$ (Fig. 7b). The streamwise balance is between the pressure gradient, advective acceleration, and bottom friction, as during maximum ebb, but local acceleration in now significant. Flow in the inlet throat decelerates, while flow along the shoreline outside the inlet is beginning to accelerate (Fig. 7d). Streamwise 

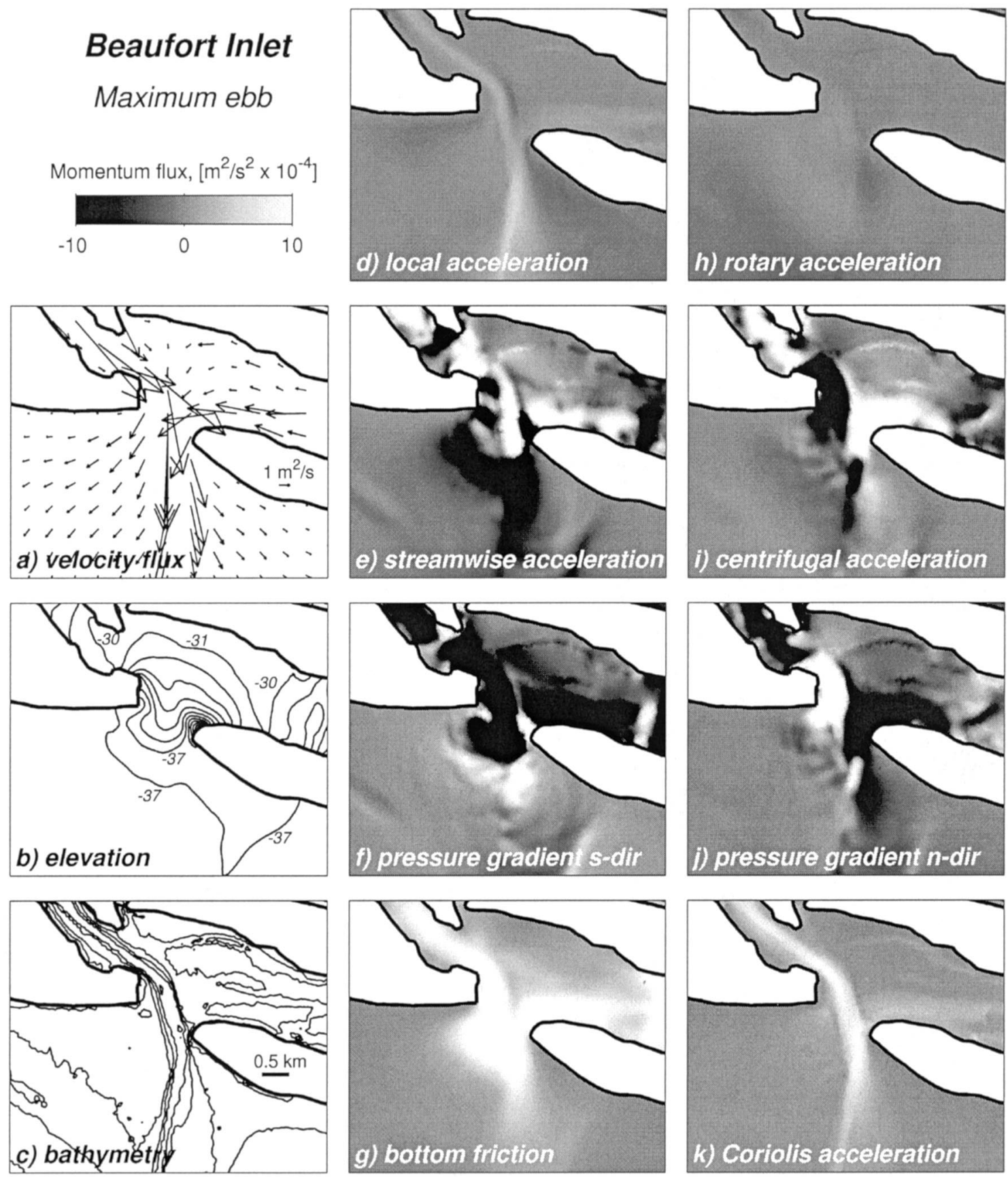

FIG. 6. Circulation and momentum balances for Beaufort Inlet at maximum ebb: (a) velocity flux (depth-averaged velocity multiplied by total water column) vectors interpolated onto uniform 425-m grid for clarity, (b) free-surface elevation with 1$\mathrm{cm}$ contour intervals, (c) bathymetry with 4-m contour intervals, and (d)-(k) shaded contours of individual momentum flux terms (see text for description).

positive advective acceleration along the sides of the ebb jet (light areas along the sides of the dark ebb jet in Fig. 7e) shows the transfer of momentum from the jet to the slower moving fluid in the lee of the headlands. The cross-stream balance shows little contribution from the rotary acceleration term (Fig. 7h), indicating that although flow speeds are decreasing, flow directions re- main fairly constant in time. The cross-inlet pressure gradient and centrifugal acceleration have weakened, but they remain the primary lateral balance (Figs. 7i,j) and are modified slightly by Coriolis (Fig. 7k).

At Beaufort Inlet, the midebb elevation difference between the sound and ocean is also about $2 \mathrm{~cm}$ (Fig. $8 b$ ), and flow in the main channel is strongly deceler- 


\section{Idealized Inlet}
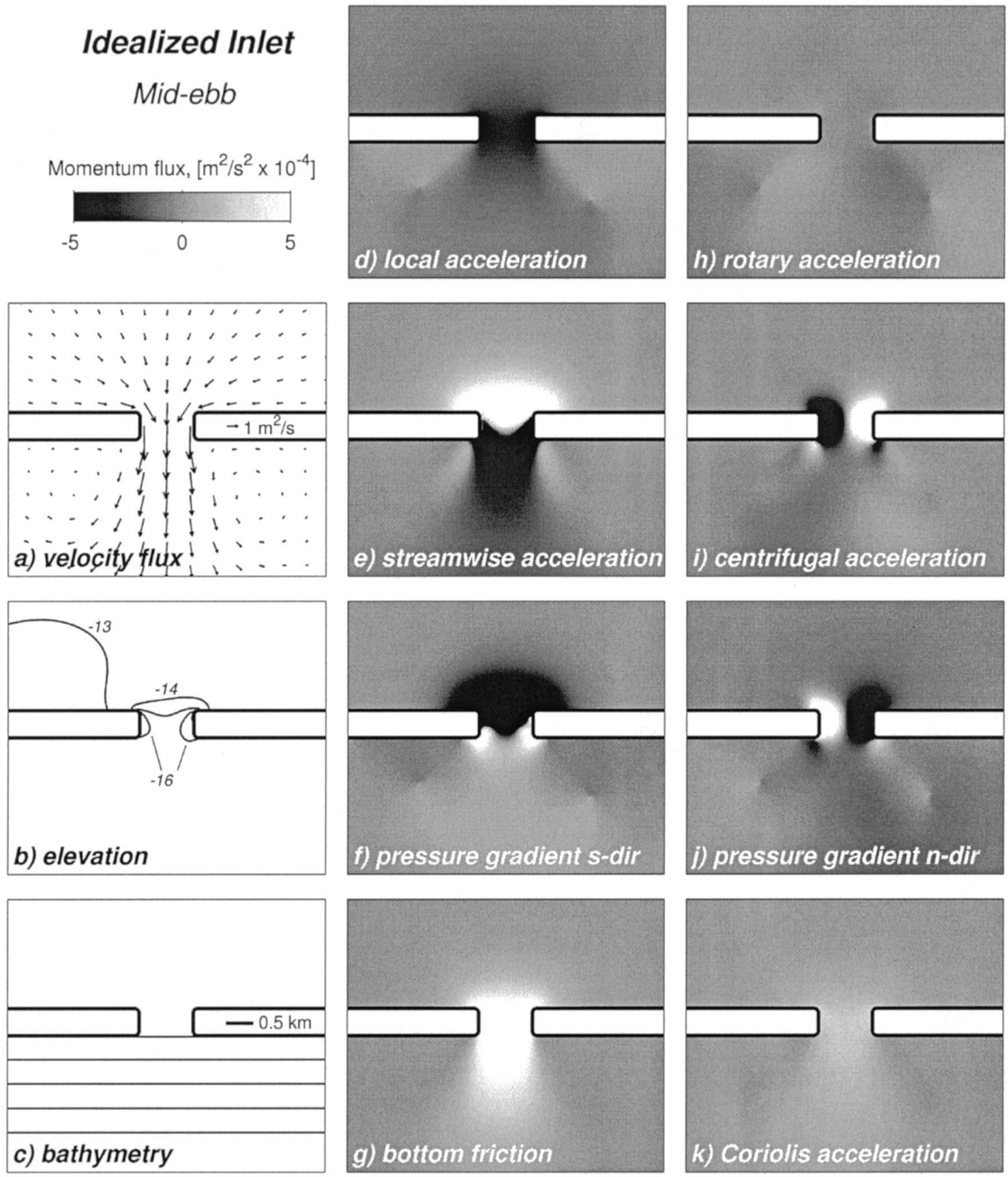

FIG. 7. Circulation and momentum balances for idealized inlet at midebb: (a) velocity flux (depth-averaged velocity multiplied by total water column) vectors interpolated onto uniform $425-\mathrm{m}$ grid for clarity, (b) free-surface elevation with 1-cm contour intervals, (c) bathymetry with 1-m contour intervals, and (d)-(k) shaded contours of individual momentum flux terms (see text for description).

ating (Fig. 8d). As in the idealized inlet, flow directions are fairly stationary (Fig. 8h). Streamwise advective accelerations have diminished significantly in magnitude from maximum ebb, but the spatial patterns remain (Fig. 8e). The large-scale offshore pressure gradient shifts prior to slack at the inlet, and high momentum flow in the outer ebb jet is decelerating under this far-field adverse pressure gradient. Along the shore outside the inlet, flow is shielded from the ebb jet by the headland features. Because of its prior weak ebb-directed momentum this fluid has already changed to a flood direction acting under the favorable offshore pressure gra- 

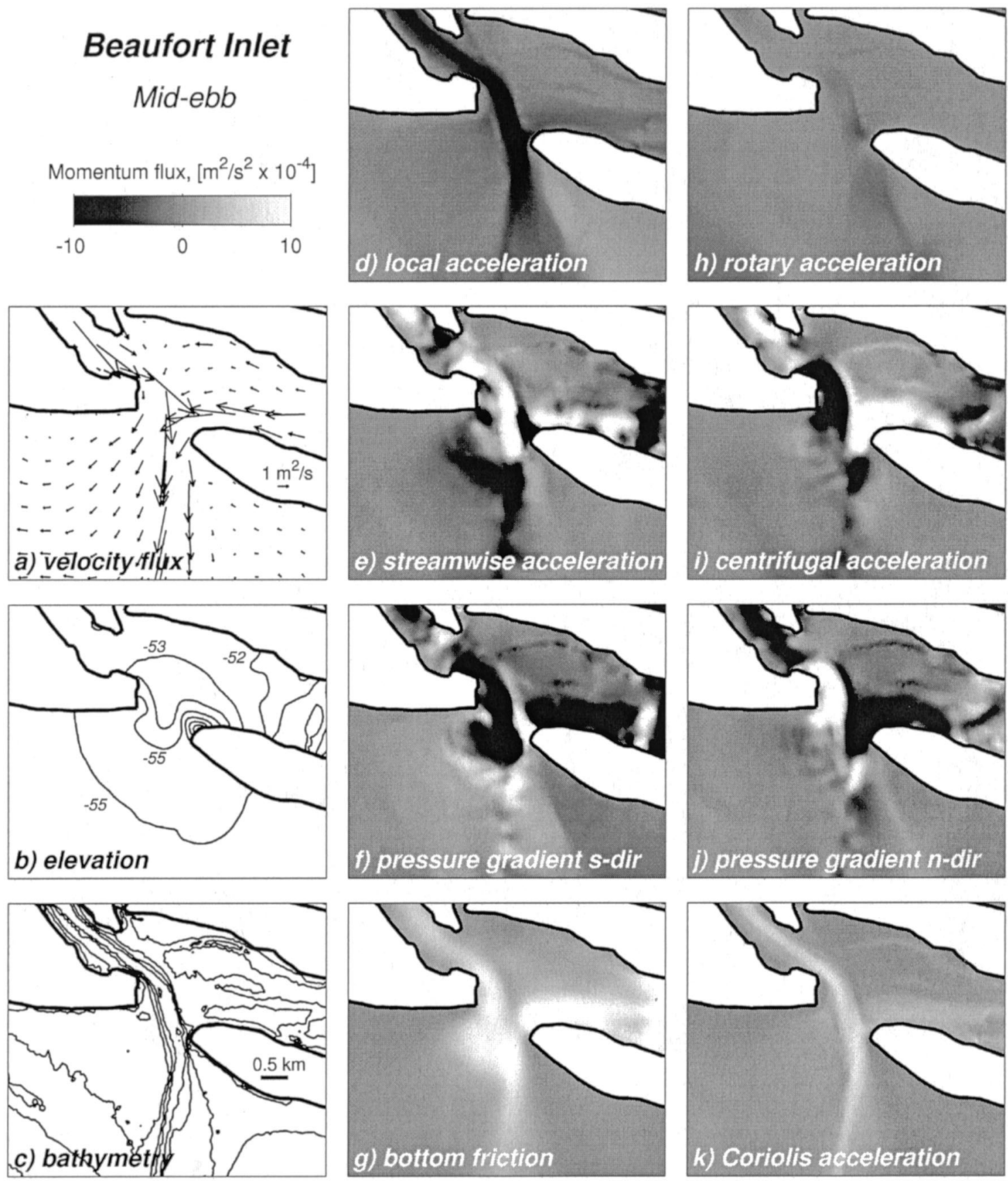

FIG. 8. Circulation and momentum balances for Beaufort Inlet at midebb: (a) velocity flux (depth-averaged velocity multiplied by total water column) vectors interpolated onto uniform 425-m grid for clarity, (b) free-surface elevation with 1-cm contour intervals, (c) bathymetry with 4-m contour intervals, and (d)-(k) shaded contours of individual momentum flux terms (see text for description).

dient (Fig. 8f). This effect is most evident east of the inlet (Fig. 8a) because of the relatively acute angle between the eastern headland and the ebb jet.

\section{c. Slack before flood}

At the start of flood the idealized inlet pressure gradient between the sound and ocean has switched, with the ocean about $3 \mathrm{~cm}$ higher than the sound (Fig. 9b). Flow within the inlet and along the ocean shore has begun to move toward the sound (Fig. 9a). These alongshore flows on the ocean side have gained sufficient strength to "pinch off" the high-momentum ebb jet that contains water ejected from the sound during the previous ebb and continues to move offshore. The jet does 


\section{Idealized Inlet}
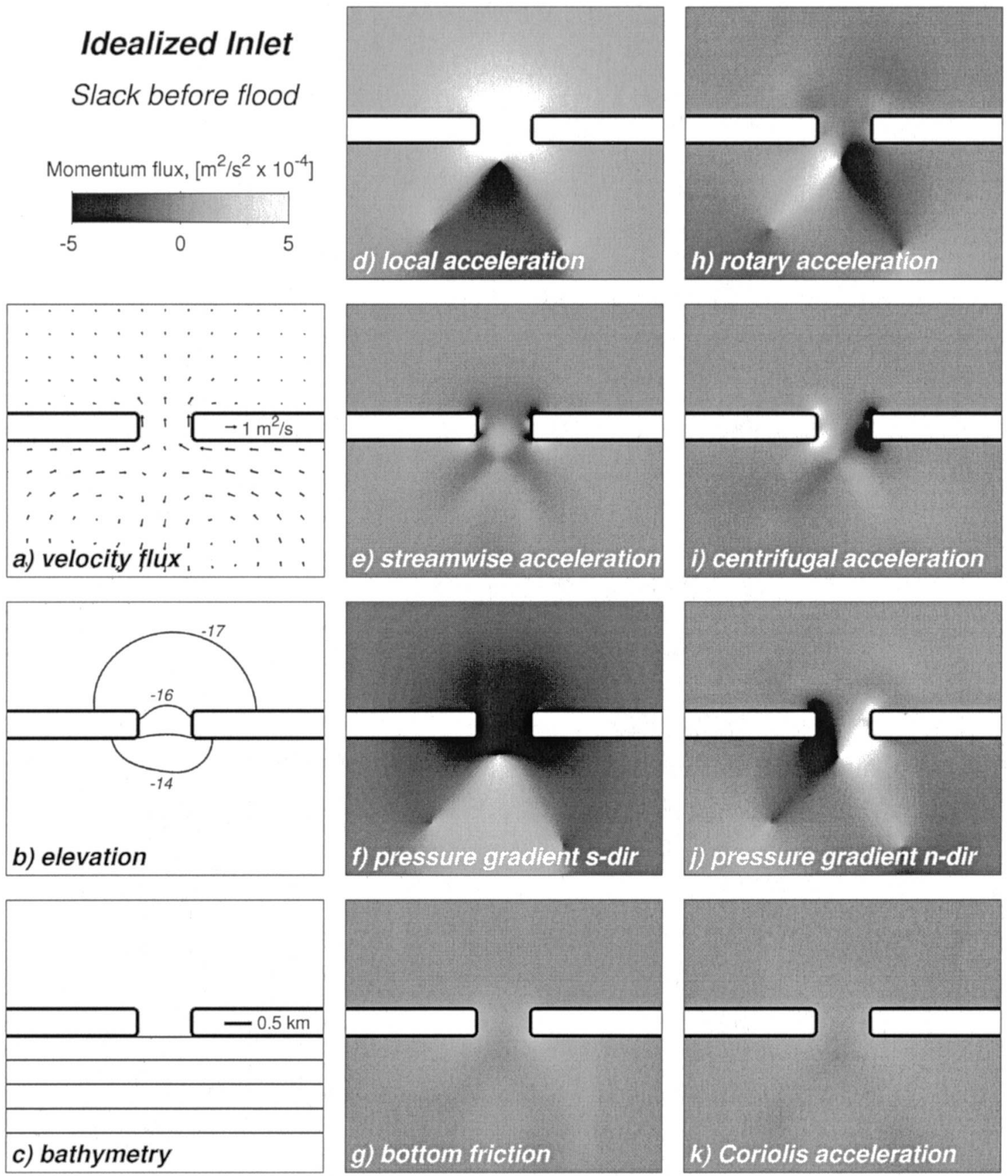

FIG. 9. Circulation and momentum balances for idealized inlet at slack before flood: (a) velocity flux (depth-averaged velocity multiplied by total water column) vectors interpolated onto uniform $425-\mathrm{m}$ grid for clarity, (b) free-surface elevation with 1 $\mathrm{cm}$ contour intervals, (c) bathymetry with 1-m contour intervals, and (d)-(k) shaded contours of individual momentum flux terms (see text for description).

not spin down completely under the influence of bottom friction, as a free jet would. Instead, the streamwise pressure gradient turns from favorable to adverse and acts to "brake" the flow (Fig. 9f), and this results in negative local acceleration (Fig. 9d). Along the sides, the ebb momentum is sufficiently weak that the time required to "brake" the flow is less than that for the jet, and therefore these areas switch to flood and positive local acceleration sooner. Bottom friction and streamwise acceleration contribute little to the streamwise balance.

In the normal direction, the primary balance is between local rotary acceleration and normal direction pressure gradient (Figs. 9h,j). Flow direction on the west 
side is changing cyclonically (turning toward the inlet) yielding positive rotary acceleration, while anticyclonic direction change on the east side (also toward inlet) gives negative rotary acceleration. Very close to the headland tips centrifugal acceleration remains important; note that the signs have switched from ebb on both sides reflecting the sign change in $R_{s}$, as leftward (rightward) curvature switches to rightward (leftward) curvature on the east (west) side.

At Beaufort Inlet the situation is somewhat more complex. At the start of flood, the offshore pressure gradient has acted long enough for the flow along the eastern shore outside the inlet to have a clear flood direction, but offshore west of the inlet flood flow is just beginning (Fig. 10a). High momentum fluid in the ebb jet continues to spin down (Fig. 10d) southwest of the inlet under the "braking" influence of the adverse pressure gradient (Fig. 10f). Inside the inlet on the west side, the velocities have turned to a flood orientation; here the flow is being fed by the east side sound (Fig. 10a). This may be a significant mechanism for cross-inlet exchange at Beaufort Inlet (see discussion). The tides in this system behave as a damped quasi-progressive wave on both estuarine sides of the inlet, however the differing geometry and depths on the two sides produce different damping and phase lags. The sound bathymetry on the east side is shallower compared to the deeper dredged west side, which results in stronger attenuation and retarding of the tide on the east side relative to the west (see Fig. 4 in Luettich et al. 1999). The differences in sound side lateral phasing drives a lateral exchange across the inlet near slack. Model runs with uniform depth $(h=7 \mathrm{~m})$ gave nearly symmetric behavior.

The normal direction momentum balance is dominated by the rotary acceleration and pressure gradient terms (Figs. 10h,j). The rotary acceleration is largest in the navigation channel where flow is turning anticyclonically toward the inlet. The normal direction pressure gradient is driving this direction change. The centrifugal acceleration is generally small except at the headland tips (Fig. 10i), and as with the idealized inlet the cross-stream momentum balance is dominated by linear terms.

\section{d. Coriolis}

The role of Coriolis in the time-dependent momentum balances is subtle and merits a more complete discussion. To assess the relative importance of Coriolis versus centrifugal acceleration in the cross-stream momentum balance, we form a "curvature" Rossby number Ro, defined in the streamline coordinate system as

$$
\text { Ro }=\left|\frac{U_{s}^{2}}{R_{s}} / f U_{s}\right|=\left|\frac{U_{s}}{f R_{s}}\right| .
$$

Model momentum fields were used to directly compute Ro at both inlets for three phases of the tide (Fig. 11).
At stronger tide phases (Figs. 11a-d) centrifugal accelerations are greater than Coriolis at both inlets (Ro $>$ 1 ), particularly close to the headlands. There is more than two orders of magnitude difference in Ro across both inlets during maximum ebb $(>20$ near headland tips, and approaching zero at the inlet centers). Most of this variability is from the radii of curvature, which range from tens of meters near the headland tips to near infinity at inlet centers where the streamlines are almost straight. During the brief $(<1 \mathrm{~h})$ period around slack tide (see Figs. 11e,f) Rossby numbers are $O(1)$ or less throughout most of the domain. For all tide phases Ro sharply decreases with distance from the inlet, except within the transient tidal eddies. In comparison to the idealized inlet, Beaufort Inlet shows considerable smallscale spatial structure associated with flow curvature from irregular bathymetry.

Ro time series from both inlets (particularly the symmetric idealized inlet) indicate that the time period that Coriolis is dynamically important is too short to cause large asymmetries in the flow field. Even during the brief periods at slack when Coriolis is larger than centrifugal acceleration, the $n$-direction momentum balance is still not geostrophic because this also coincides with the period when local accelerations are significant. This point is clear from the highly symmetric circulation fields (Fig. 3) and Ro contours for the idealized inlet (Fig. 11), but less so for the natural inlet as the effects of bathymetric asymmetries obscure those potentially caused by Coriolis. Conceptually one would expect that Coriolis would act constructively or destructively to the centrifugal acceleration and, for example, shift the position of maximum surface elevation across the inlet. Such an effect is very slight for the idealized inlet, while at Beaufort Inlet the effect is entirely obscured by asymmetries due to irregular bathymetry.

\section{Discussion}

Dynamical balances for the remainder of the tidal cycle (not shown because of space considerations) closely follow those described above for the ebb. The temporal evolution of the momentum fields shows that the balances oscillate between two dynamical states. At maximum flow and throughout much of the tidal cycle, the nonlinear terms and pressure gradients dominate the momentum balance, whereas near slack the balance is dominated by the linear terms. During the transition between the two end states, local acceleration is important, but the rotary acceleration is small as most of the flow direction change occurs during a brief period around slack.

As noted in section 3, the idealized inlet and Beaufort Inlet have different wave characteristics (standing vs quasi-progressive) and we wondered what effect this has on inlet momentum balances. We reran the idealized inlet model with a Sommerfeld type radiation condition in the sound (to mimic the effect of the extensive sound regions surrounding Beaufort Inlet), which yielded near- 

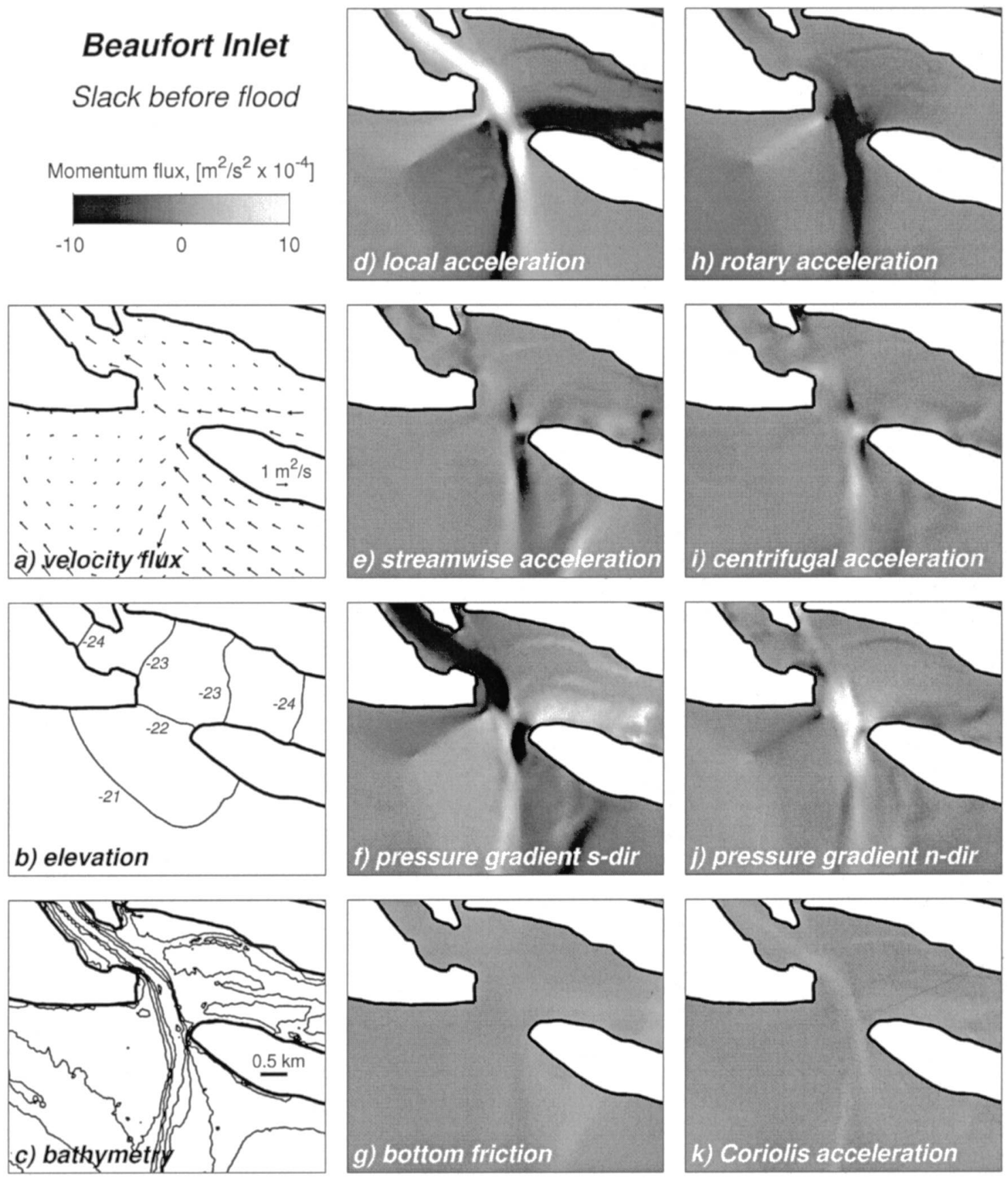

FIG. 10. Circulation and momentum balances for Beaufort Inlet at slack before flood: (a) velocity flux (depth-averaged velocity multiplied by total water column) vectors interpolated onto uniform 425-m grid for clarity, (b) free-surface elevation with 1cm contour intervals, (c) bathymetry with 4-m contour intervals, and (d)-(k) shaded contours of individual momentum flux terms (see text for description).

ly pure progressive wave behavior. We then adjusted the forcing to obtain about $1 \mathrm{~m} \mathrm{~s}^{-1}$ maximum velocities in the inlet, and recomputed the corresponding momentum balances. There was very little difference in the spatial patterns of momentum between the standing and progressive waves, and it became clear that local inlet hy- draulics dominate over broader-scale shelf-sound wave behavior. Model runs with differing inlet aspect ratios (i.e., length and width) showed significantly different balances within the inlets, and these results are reported along with a dynamical classification scheme in a companion paper (Hench et al. 2002). 

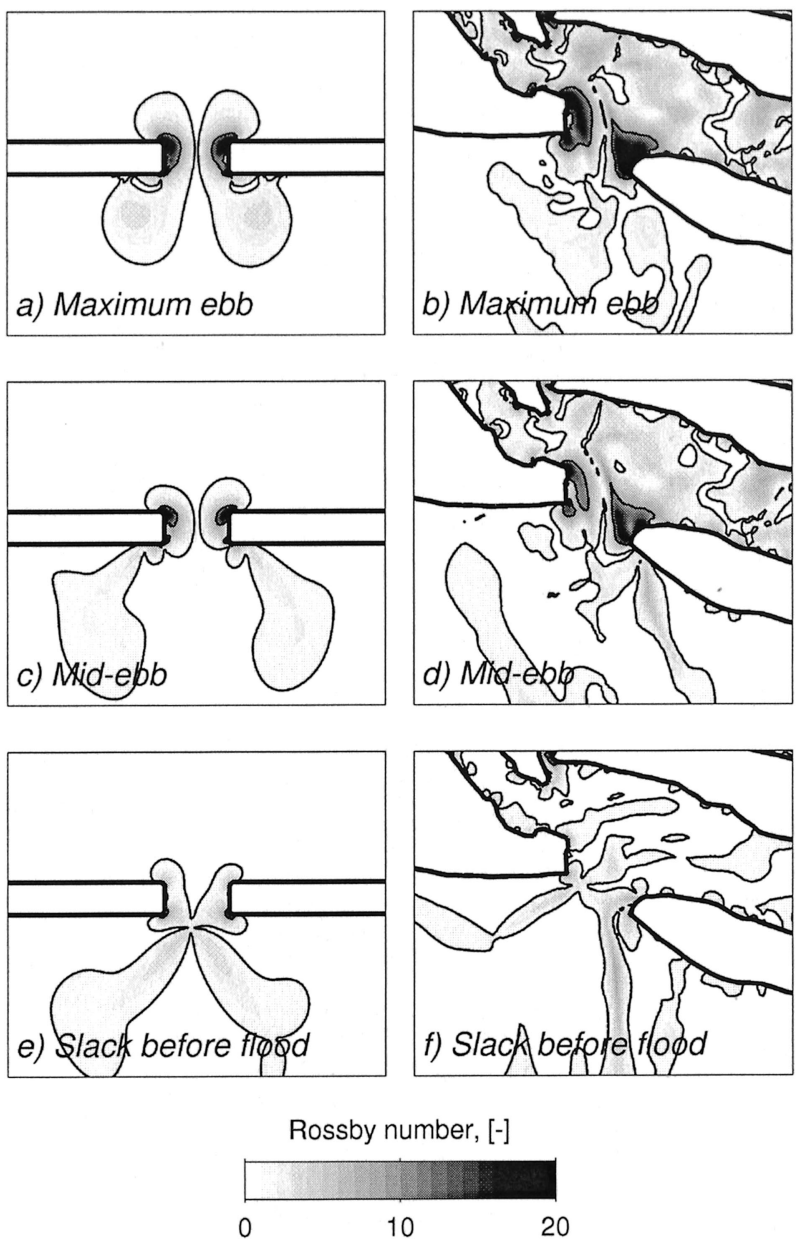

FIG. 11. (a)-(f) Rossby number (defined as Ro $=\left|U_{s} / f R_{s}\right|$ ) shaded contours at various phases of the tide. The solid black contour lines indicate $\mathrm{Ro}=1$ and $\mathrm{Ro}=10$.

\section{a. Transient eddies and ocean-sound exchange}

The transient eddies which form behind the headland features at shallow inlets have been described as phase eddies to distinguish them from recirculations due to pure flow separation (Black and Gay 1987). These authors proposed that the phase lags between flow in the center of the inlet and the inlet sides were due to enhanced bottom friction on the shallow ebb shoals and reduced bottom friction in the deep main channel. The results from the idealized inlet in this study (with uniform alongshore bathymetry) suggest that spatial differences in bottom friction are not needed to generate these phase lags. Rather the generation mechanism for the phase eddies is "headland sheltering" of momentum (from the ebb jet) along the sides of the inlet. At maximum ebb, the ebb jet contains strong $U_{s} \partial U_{s} / \partial s$ (locally balanced by bottom friction), while on both sides of the jet, $U_{s} \partial U_{s} / \partial s$ is weak. Toward the end of ebb, the jet does not spin down completely under the influence of bottom friction, as a free jet would. Instead, the large- scale offshore streamwise pressure gradient $g \partial \eta / \partial s$ turns from favorable to adverse and acts to "brake" the flow, resulting in negative $\partial U_{s} / \partial t$. Along the jet sides, the flow is sufficiently weak that the time required to "brake" the flow is less than that for the jet, and therefore switches sooner to flood and positive $\partial U_{s} / \partial t$. The difference in the time it takes the flood adverse pressure gradient to turn the tide from the jet relative to the sides is the source of the phase lag. This same general behavior was seen at Beaufort Inlet, but the offset inlet headlands acting in conjunction with phase lags within the sound produced an asymmetric flood flow pattern, with the eastern side of the inlet flooding first.

The transient eddies are largely responsible for inlet exchange of sound water with ocean water, as illustrated with modeled particle trajectories in Fig. 12. Early in the flood the offshore streamwise pressure gradient drives flow along the ocean shoreline and into the inlet (Figs. 12e,f). While the ebb jet continues to spin down offshore, ocean water (which may be "new" or a diluted mixture of estuarine and ocean water from previous ebbs) flows behind it and isolates water contained in the ebb jet. Therefore one would expect initial flood water at an inlet to have a higher salinity than found at midflood, with salinities increasing again during late flood. This generic picture is somewhat altered at Beaufort Inlet as ocean water preferentially enters the east side of the inlet.

\section{b. Implications for cross-inlet exchange}

While it is clear that significant mixing and exchange occurs at tidal inlets, as described above, the results of this study show that this notion should be qualified in the cross-inlet direction. During the stronger phases of the tide, the primary lateral balance within the inlet is between centrifugal acceleration and lateral pressure gradients. The centrifugal acceleration is greatest near the headland tips, and approaches zero at the inlet center (where streamlines become straight and $R_{s}$ approaches infinity). The water surface adjusts by dipping near the headland tips and sloping upward toward the inlet center, forming a dome of water across the inlet. The significance of this is that the sign of the lateral pressure gradient changes from one side of the inlet to the other (i.e., opposing pressure gradients push against each other much in the same way a solid boundary opposes the centrifugal acceleration in the case of a river bend). Since the barotropic pressure gradient is uniform with depth, these forces constrain flow to remain on the side of the inlet that it entered the inlet on. We expect this "dynamical wall effect" to significantly restrict lateral mixing within an inlet. Modeled inlet drifter trajectories for the idealized inlet and Beaufort Inlet clearly illustrate this effect as they are turned around the headland features, but not across the inlets (see Figs. $12 \mathrm{~g}-\mathrm{j}$ ). Previous observed (Churchill et al. 1999) and model (Luettich et al. 1999; Brown et al. 2000) drifter studies also exhib- 

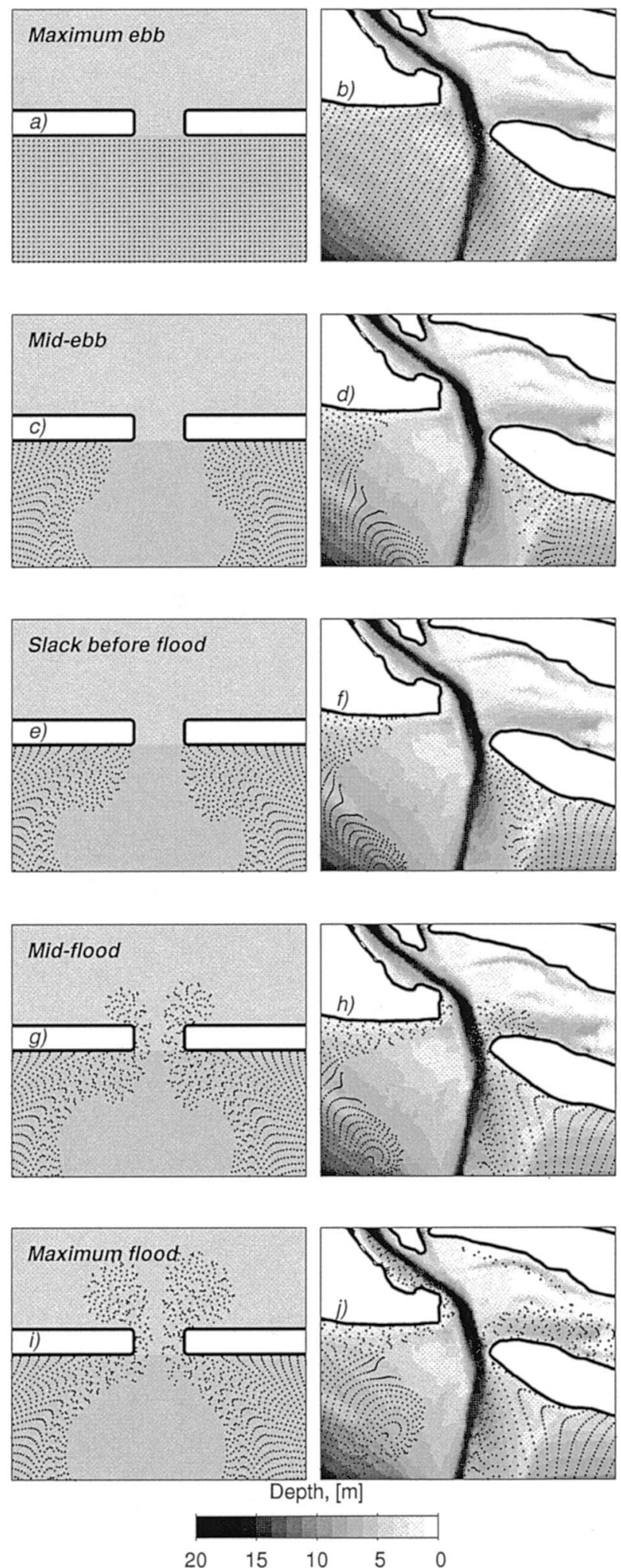

FIG. 12. Lagrangian particle trajectories over a half tidal cycle for (a), (c), (e), (g), (i) the idealized inlet and (b), (d), (f), (h), (j) Beaufort inlet. Particles were released at maximum ebb, and positions were computed using a particle tracking model (see Baptista et al. 1984; Foreman et al. 1992). ited minimal cross-inlet exchange during the stronger tidal phases and further support this physical explanation.

In fully three-dimensional flow, curvature generates a secondary flow pattern (in the $n-z$ plane) caused by an imbalance between lateral barotropic pressure gradients and centrifugal accelerations [see related work on river bends (Bathurst et al. 1977; Kalkwijk and Booij 1986), headlands (Geyer 1993; Chant and Wilson 1997), and curved tidal channels (Seim and Gregg 1997)]. As noted above, the lateral barotropic pressure gradients are constant with depth, but in three dimensions, centrifugal accelerations vary over the water column (maximum near the surface and decreasing with depth) because of the depth dependence of the streamwise velocity. As the flow turns around a bend or headland (and assuming no stratification), water near the surface has a relative excess of centrifugal acceleration and a secondary flow is driven toward the outside of the curve. Near bottom a deficit in centrifugal acceleration relative to the pressure gradient drives secondary flow toward the inside of the curve. At inlets the circulation is more complex as two flows curve around opposing headlands; centrifugal forces would tend to force both surface (bottom) flows away from (toward) both headlands. One would expect this secondary circulation to produce convergence and downwelling at the inlet center, divergence along the bottom, and upwelling at the inlet sides. This lateral mixing mechanism is entirely barotropic and would occur on both ebb and flood.

In a two-dimensional model, these secondary cells cannot form because of the depth independence of the horizontal velocities, but have been shown to be $10 \%-$ $20 \%$ of the streamwise flow in river bends (Bathurst et al. 1977) and near headlands (Geyer 1993). We expect similar secondary flow magnitudes for the inlets in this study and estimated the maximum secondary circulation (due to barotropic centrifugal acceleration alone) as $U_{n, \max } \approx 6 U_{s} H / R_{s}$ (Geyer 1993). If $R_{s} \sim 500 \mathrm{~m}, H \sim$ $10 \mathrm{~m}$, and $U_{s} \sim 1 \mathrm{~m} \mathrm{~s}^{-1}$, then $U_{n \text { max }}$ is about $0.12 \mathrm{~m}$ $\mathrm{s}^{-1}$. Assuming that a water parcel traveling through an inlet would have a streamwise trajectory length of 1000 $\mathrm{m}$, the travel time through the secondary circulation zone would be of order $1000 \mathrm{~s}$. Therefore an estimate of the lateral distance traveled due to secondary circulation while passing through these inlets is $120 \mathrm{~m}$ or about $25 \%$ of the half-inlet width. This scaling argument suggests lateral vertical shear from secondary circulation can reduce lateral density gradients, or partially mix lateral gradients of passive scalars (e.g., pollutants, dissolved oxygen, biota) on each side of the inlet (but not across the inlet centerline). It remains for three-dimensional studies to fully examine this effect.

Perhaps somewhat counterintuitively, the greatest amount of cross-inlet exchange may occur near slack tidal phases when the pressure gradient-centrifugal acceleration balance is nearly absent. In natural inlets there are usually lateral phase differences within the sound 
and offshore due bathymetric asymmetries (unique to each system). If this is the case, near slack, currents on one side of the inlet will change direction before the other and may flow across the inlet (as seen in Fig. 10a). This mechanism is not present in symmetric idealized inlets and therefore has not been identified previously. At Beaufort Inlet, water tends to flow from east to west across the inlet at the start of flood, and provides a mechanism for transporting material to the sound west of the inlet. The addition of wind forcing or lateral baroclinic pressure gradients may make cross inlet exchange during slack tidal phases particularly effective. However, as the tide gains strength, these cross-inlet pathways will again be "walled off" by the dominant pressure gradient-centrifugal acceleration balance and sharply diminish the possibility for cross-inlet exchange.

Inlet morphology may also play a role in determining the amount of cross channel exchange and the fate of water entering an inlet. Our model results indicate that the pressure gradient-centrifugal acceleration balance sharply diminishes with distance from the headland features. This distance scales with the inertial radius $r=$ $U_{s} / f$ (Hench et al. 2002). If the bottom is flat and the geometry is simple (as in the idealized inlet), cross-inlet mixing may take place in zones inshore or offshore of the inlet where the "wall effect" is dynamically small. However, many natural inlets (including Beaufort Inlet) have channels that bifurcate on the sound side. In these cases channelization may preserve two distinct water masses inside the inlet (see Fig. 12j).

\section{c. Conclusions}

An analysis of transient momentum balances has elucidated the dynamics, circulation patterns, and exchange mechanisms at shallow barotropic tidal inlets. Circulation computed with high-resolution models was used to directly evaluate the contribution of each term in the momentum equations to the overall momentum balance. Transformation of the time-dependent, frictional, fully nonlinear $x-y$ shallow water equations into an $s-n$ coordinate system greatly simplifies interpretation of the dynamics. This set of equations appears to be the simplest dynamics that retains all the essential physics; inlet circulation is at least a two-dimensional transient nonlinear problem.

The temporal evolution of momentum indicates that inlet momentum balances oscillate between two dynamical states. During the stronger tidal phases, the streamwise balance is between pressure gradient and streamwise acceleration in the sink region, and between streamwise deceleration and bottom friction in the ebb jet. Cross-stream balances are between centrifugal acceleration and normal direction pressure gradients. Near slack, the dynamics are nearly linear with streamwise pressure gradients balancing local acceleration, and normal direction pressure gradients balancing rotary accleration.
Spatial patterns in the momentum balance terms show the dynamics can vary dramatically over subkilometer distances. High grid resolution revealed small-scale features, such as localized flow separation zones, and the dynamical comparison between idealized and natural inlets showed the importance of topography. The inletscale momentum features in our models are in general agreement with the previous results of Imasato (1983) and Ridderinkhof (1988). However, the transient dynamical features described above were not identified in their results.

The time-dependent dynamics have direct consequences on inlet exchange during the transition between the two end states. As the tide switches from ebb to flood, the offshore spatially uniform streamwise pressure gradient acts upon a spatially variable ebb jet flow field. Low momentum fluid behind the headland features (sheltered from the ebb jet) switches to flood before the jet, and "new" ocean water floods the inlet first, while sound water in the jet continues to spin down under the "braking" influence of the adverse pressure gradient. As the tide gains strength, secondary circulation may induce lateral mixing within the inlet but not across the inlet centerline because of the "dynamical wall effect." To our knowledge the formation and relaxation of the dome of water across an inlet during a tidal cycle has not been directly observed in nature and this appears to be a case where model results precede direct measurements. The models indicate that the cross-inlet elevation differences are at least several centimeters. Given the general tendency of numerical models to underpredict sharp velocity and elevation gradients, this is probably a lower bound of what can be found in nature. If this is true, the dome should be within the detection limits of standard oceanographic pressure sensors.

The wall effect may help to explain a number of exchange and transport processes found in nature. For example, in a study of larval ingress at Beaufort Inlet, Forward et al. (1999) observed fish larvae concentrations in the sound that were an order of magnitude greater on the east side of the inlet than on the west side. If the major offshore source of these larvae was east of the inlet, the wall effect (and channelization in the sound) would confine them to the east side of the sound where their fate would be determined by the quality of nursery habitat in that part of the sound. Similar crossinlet differences in transport may occur for other plankton, as well as contaminants, nutrients, and suspended sediments.

Acknowledgments. We thank Brian Blanton and Crystal Fulcher for help setting up and running the models. The crew of the NOAA ship Whiting kindly provided 1998 bathymetric data. Discussions with John Bane, Jack Blanton, Cheryl Brown, and comments from three anonymous reviewers significantly improved the manuscript. Funding was provided by USACOE Coastal In- 
lets Research Program (CIRP), ONR (N00014-97-C6010), and NSF (OCE-0094938).

\section{APPENDIX \\ Rotation of $x-y$ Momentum Equations into an $s-n$ Coordinate System}

The use of a streamwise-normal coordinate system allows a more intuitive physical interpretation for strongly curving flow fields. Here we derive a form of the fully nonlinear transient frictional shallow water equations by transforming the familiar $x-y$ equations. Here $U(x, y, t), V(x, y, t)$, and $\eta(x, y, t)$ are computed on a fixed $x-y$ grid for all time steps. The elevation and velocity fields are used to reconstruct each term in the $x-y$ momentum equations at each node in the $x-y$ grid. Individual $x-y$ momentum terms $M_{x i}$ and $M_{y i}$ are designated as

$$
\begin{aligned}
& \underset{M_{x 1}}{\frac{\partial U}{\partial t}}+\underbrace{U \frac{\partial U}{\partial x}+V \frac{\partial U}{\partial y}}_{M_{x 2}}-\underset{M_{x 3}}{f V}+\underset{M_{x 4}}{g \frac{\partial \eta}{\partial x}} \\
& +\underbrace{\left(\frac{C_{f} \sqrt{U^{2}+V^{2}}}{H}\right) U}_{M_{x 5}}=0, \\
& \underset{M_{y 1}}{\frac{\partial V}{\partial t}}+\underbrace{U \frac{\partial V}{\partial x}+V \frac{\partial V}{\partial y}}_{M_{y 2}}+\underbrace{f U}_{M_{y 3}}+\underset{M_{y 4}}{g \frac{\partial \eta}{\partial y}} \\
& +\underbrace{\left(\frac{C_{f} \sqrt{U^{2}+V^{2}}}{H}\right) V}_{M_{y 5}}=0 .
\end{aligned}
$$

For simplicity, horizontal diffusion terms are omitted from (A1) and (A2) as they did not contribute significantly to the momentum balances of our inlet simulations. At each time step, we define a two-dimensional, orthogonal, curvilinear coordinate system such that at each grid point, one coordinate direction points in the streamwise direction $(s)$ and the other points in the across-stream or normal direction $(n)$ in the right-hand sense (see Fig. A1). The orientation of the $s-n$ coordinate system relative to the original fixed $x-y$ coordinate system, given by the streamline angle $\alpha(x, y, t)$,

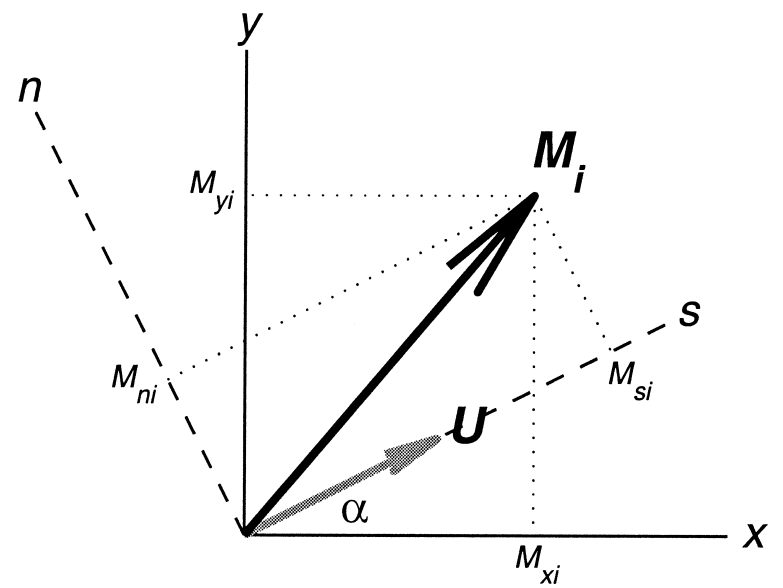

FIG. A1. Coordinate system definition sketch for the $x-y$ to $s-n$ transformation. At each time step, an $s-n$ coordinate system is established (aligned with the local velocity vector $\mathbf{U}$ at each computational node. The local axes rotation angle is $\alpha(x, y, t)$. Vector $\mathbf{M}_{i}$ represents the $i$ th force or acceleration vector in the momentum equations, with $x-y$ components $M_{x i}$ and $M_{y i}$ (e.g., $g \partial \eta / \partial x$ and $g \partial \eta / \partial y$ ), and is rotated onto the $s-n$ axes to determine local $s-n$ components $M_{s i}$ and $M_{n i}$ (e.g., $g \partial \eta / \partial s$ and $\left.g \partial \eta / \partial n\right)$.

varies so that at all points the alongstream velocity $U_{s}$ is equivalent to the speed, and the across-stream velocity $U_{n}=0$. From Fig. A1 it should be apparent that momentum terms in $s-n$ coordinates are related to those in the $x-y$ coordinate system by

$$
\begin{aligned}
& M_{s i}=M_{x i} \cos \alpha+M_{y i} \sin \alpha, \\
& M_{n i}=M_{y i} \cos \alpha-M_{x i} \sin \alpha,
\end{aligned}
$$

where the index $i=1: 5$ as in Eqs. (A1) and (A2) above. The $s-n$ velocities can be related to the $x-y$ velocity components using the same orthogonal rotation:

$$
\begin{aligned}
& U_{s}=U \cos \alpha+V \sin \alpha=\sqrt{U^{2}+V^{2}}, \\
& U_{n}=V \cos \alpha-U \sin \alpha \equiv 0 .
\end{aligned}
$$

From Eq. (A6), $\alpha=\arctan (V / U)$. Equations (A5) and (A6) can also be rearranged to express $(U, V)$ in terms of $U_{s}$ and $\alpha$ :

$$
\begin{aligned}
& U=U_{s} \cos \alpha, \\
& V=U_{s} \sin \alpha .
\end{aligned}
$$

Substituting the $x-y$ momentum terms from (A1) and (A2) into (A3) and (A4), and replacing $U$ and $V$ using (A7) and (A8), gives

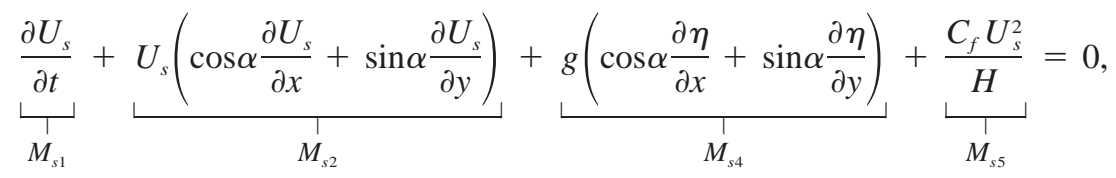




$$
\underbrace{U_{s} \frac{\partial \alpha}{\partial t}}_{M_{n 1}}+\underbrace{U_{s}^{2}\left(\cos \alpha \frac{\partial \alpha}{\partial x}+\sin \alpha \frac{\partial \alpha}{\partial y}\right)}_{M_{n 2}}+\underbrace{f U_{s}}_{M_{n 3}}+\underbrace{g\left(\cos \alpha \frac{\partial \eta}{\partial y}-\sin \alpha \frac{\partial \eta}{\partial x}\right)}_{M_{n 4}}=0 .
$$

With this transformation $M_{\mathrm{s} 3}$ and $M_{n 5}$ are zero by definition (i.e., bottom friction acts entirely in the streamwise direction, and Coriolis only in the normal direction). To complete the transformation, derivatives in the $x-y$ and $s-n$ coordinate systems are related by the chain rule:

$$
\begin{aligned}
& \frac{\partial}{\partial x}=\frac{\partial}{\partial s} \frac{\partial s}{\partial x}+\frac{\partial}{\partial n} \frac{\partial n}{\partial x}=\cos \alpha \frac{\partial}{\partial s}-\sin \alpha \frac{\partial}{\partial n}, \\
& \frac{\partial}{\partial y}=\frac{\partial}{\partial s} \frac{\partial s}{\partial y}+\frac{\partial}{\partial n} \frac{\partial n}{\partial y}=\sin \alpha \frac{\partial}{\partial s}+\cos \alpha \frac{\partial}{\partial n},
\end{aligned}
$$

where $\partial s / \partial x=\cos \alpha, \partial s / \partial y=\sin \alpha, \partial n / \partial x=-\sin \alpha$, and $\partial n / \partial y=\cos \alpha$ from Fig. A1. The final relationship needed is $\partial \alpha / \partial s=1 / R_{s}$, where $R_{s}(x, y, t)$ is the streamwise radius of curvature (cf. Gill 1982; Munson et al. 1994), with curvature to the left assumed positive. Expanding the spatial derivatives in (A9) and (A10) with the chain rule, substituting in the expression for $R_{s}$, and simplifying yields the momentum equations in $s-n$ coordinates

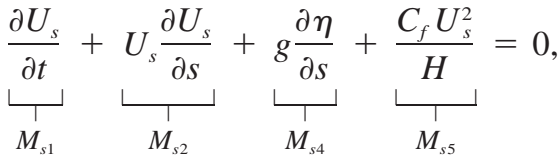

$$
\begin{aligned}
& \underbrace{U_{s} \frac{\partial \alpha}{\partial t}}_{M_{n 1}}+\underbrace{\frac{U_{s}^{2}}{R_{s}}}_{M_{n 2}}+\underbrace{f U_{s}}_{M_{n 3}}+\underset{M_{n 4}}{\underbrace{}_{\mid} \frac{\partial \eta}{\partial n}}=0 .
\end{aligned}
$$

(A similar procedure can be followed to obtain the $s-$ $n$ form of the continuity equation, which is $\partial \eta / \partial t+$ $\partial U_{s} H / \partial s+U_{s} H \partial \alpha / \partial n=0$.) Note that once values of the $x-y$ momentum terms are known, along with the streamline angles, the values of the $s-n$ momentum terms can be computed directly using (A3) and (A4). Thus the right-hand sides of (A3)-(A4) are actually calculated and the left-hand sides of (A3)-(A4) are the physical quantities to interpret. This methodology is useful because one retains the computational ease of working in a standard $x-y$ coordinate system and with a simple transform gains the interpretational advantages of the $s-n$ coordinate system.

\section{REFERENCES}

Baptista, A. M., E. E. Adams, and K. D. Stolzenbach, 1984: EulerianLagrangian analysis of pollutant transport in shallow water. R. M. Parsons Lab. Tech. Rep. 296, Massachusetts Institute of Technology, $140 \mathrm{pp}$.

Bathurst, J. C., C. R. Thorne, and R. D. Hey, 1977: Direct measure- ments of secondary currents in river bends. Nature, 269, 504506.

Black, K. P., and S. L. Gay, 1987: Eddy formation in unsteady flows. J. Geophys. Res., 92, 9514-9522.

Blanton, J. O., F. E. Werner, A. Kapolnai, B. O. Blanton, D. Knott, and E. L. Wenner, 1999: Wind-generated transport of fictitious passive larvae into shallow tidal estuaries. Fish. Oceanogr., 8 (Suppl.), 210-223.

Brown, C. A., G. A. Jackson, and D. A. Brooks, 2000: Particle transport through a narrow tidal inlet due to tidal forcing and implications for larval transport. J. Geophys. Res., 105, $24141-$ 24156.

Carr, E. E., and N. C. Kraus, 2002: Federal inlets database. Proc. 2002 National Conf. Beach Preservation Technology, Biloxi, MS, Florida Shore and Beach Preservation Association, 119126.

Chadwick, D. B., and J. L. Largier, 1999a: The influence of tidal range on the exchange between San Diego Bay and the ocean. J. Geophys. Res., 104, 29 885-29 899.

$\longrightarrow$, and $\longrightarrow, 1999 \mathrm{~b}$ : Tidal exchange at the bay-ocean boundary. J. Geophys. Res., 104, 29 901-29 924.

Chant, R. J., and R. E. Wilson, 1997: Secondary circulation in a highly stratified estuary. J. Geophys. Res., 102, 23 207-23 215.

Chao, S.-Y., 1990: Tidal modulation of estuarine plumes. J. Phys. Oceanogr., 20, 1115-1123.

Churchill, J. H., J. O. Blanton, J. L. Hench, R. A. Luettich Jr., and F. E. Werner, 1999: Flood tide circulation near Beaufort Inlet, North Carolina: Implications for larval recruitment. Estuaries, 22, 1057-1070.

Cromwell, J. E., 1973: Barrier coast distribution: a worldwide survey. Barrier Islands, M. L. Schwartz, Ed., Dowdon, Hutchinson and Ross, 407-408.

Crowder, L. B., and F. E. Werner, Eds., 1999: Fisheries oceanography of the estuarine-dependent fishes of the South Atlantic Bight: An interdisciplinary synthesis of SABRE (South Atlantic Bight Recruitment Experiment). Fish. Oceanogr., 8 (Suppl. 2), 252 pp.

Fenster, M. R., and R. Dolan, 1996: Assessing the impact of tidal inlets on adjacent barrier island shorelines. J. Coastal Res., 12, 294-310.

Foreman, M. G. G., A. M. Baptista, and R. A. Walters, 1992: Tidal model studies of particle trajectories around a shallow coastal bank. Atmos.-Ocean, 30, 43-69.

Forward, R. B., Jr., and Coauthors, 1999: Transport of fish larvae through a tidal inlet. Fish. Oceanogr., 8 (Suppl.), 153-172.

Geyer, W. R., 1993: Three-dimensional tidal flow around headlands. J. Geophys. Res., 98, 955-966.

_- , and R. P. Signell, 1992: A reassessment of the role of tidal dispersion in estuaries and bays. Estuaries, 15, 97-108.

Gill, A. E., 1982: Atmosphere-Ocean Dynamics. Academic Press, $662 \mathrm{pp}$.

Hench, J. L., B. O. Blanton, and R. A. Luettich Jr., 2002: Lateral dynamic analysis and classification of barotropic tidal inlets. Cont. Shelf Res., 15, 2615-2631.

Imasato, N., 1983: What is tide-induced residual current? J. Phys. Oceanogr., 13, 1307-1317.

—_ 1987: Application limit of tide-induced residual current theory-Criticism of previous studies on a circular tide-induced residual current. J. Oceanogr. Soc. Japan, 43, 319-331.

_ - S. Fujio, Q. Zhang, T. Awaji, and K. Akitomo, 1994: Threedimensional numerical experiments on tidal exchange through a narrow strait in a homogeneous and a stratified sea. J. Oceanogr., 50, 119-139. 
Kalkwijk, J. P. T., and R. Booij, 1986: Adaptation of secondary flow in nearly-horizontal flow. J. Hydraul. Res., 24, 19-37.

Kapolnai, A., F. E. Werner, and J. O. Blanton, 1996: Circulation, mixing, and exchange processes in the vicinity of tidal inlets: A numerical study. J. Geophys. Res., 101, 14 253-14 268.

Kjerfve, B., and J. A. Proehl, 1979: Velocity variability in a crosssection of a well-mixed estuary. J. Mar. Res., 37, 409-418.

Luettich, R. A., Jr., J. J. Westerink, and N. W. Scheffner, 1992: ADCIRC: An advanced three-dimensional model for shelves, coasts, and estuaries. Report 1: Theory and methodology of ADCIRC 2DDI and ADCIRC 3-DL. Dredging Research Program Tech. Rep. DRP-92-6, Coastal Engineering Research Center, U. S. Army Corps of Engineers, Waterways Experiment Station, 141 pp.

, J. L. Hench, C. W. Fulcher, F. E. Werner, B. O. Blanton, and J. H. Churchill, 1999: Barotropic tidal and wind driven larvae transport in the vicinity of a barrier island inlet. Fish. Oceanogr., 8 (Suppl.), 190-209.

Militello, A., and G. A. Zarillo, 2000: Tidal motion in a complex inlet and bay system, Ponce de Leon Inlet, Florida. J. Coastal Res., 16, 840-852.

Munson, B. R., D. F. Young, and T. H. Okiishi, 1994: Fundamentals of Fluid Mechanics. John Wiley and Sons, 893 pp.

Parker, B. B., 1991: The relative importance of the various nonlinear mechanisms in a wide range of tidal interactions (review). Tidal Hydrodynamics, B. B. Parker, Ed., John Wiley and Sons, 237268.

Pietrafesa, L. J., J. O. Blanton, J. D. Wang, V. Kourafalou, T. N. Lee, and K. A. Bush, 1985: The tidal regime in the South Atlantic Bight. Oceanography of the Southeastern U.S. Continental Shelf, L. P. Atkinson et al., Eds., Coastal and Estuarine Studies, Vol. 2, Amer. Geophys. Union, 63-76.
Redfield, A. C., 1958: The influence of the continental shelf on the tides of the Atlantic coast of the United States. J. Mar. Res., 17, 432-448.

Ridderinkhof, H., 1988: Tidal and residual flows in the Western Dutch Wadden Sea, I: Numerical model results. Netherlands J. Sea Res., 22, 1-21.

Seim, H. E., and M. C. Gregg, 1997: The importance of aspiration and channel curvature in producing strong vertical mixing over a sill. J. Geophys. Res., 102, 3451-3472.

Sheng, Y. P., S. Peene, and E. Yassuda, 1996: Circulation and transport in Sarasota Bay, Florida: The effect of tidal inlets on estuarine circulation and flushing quality. Mixing in Estuaries and Coastal Seas, C. Pattiaratchi, Ed., Coastal and Estuarine Studies, Vol. 50, Amer. Geophys. Union, 184-210.

Signell, R. P., and W. R. Geyer, 1991: Transient eddy formation around headlands. J. Geophys. Res., 96, 2561-2575.

_- and B. Butman, 1992: Modeling tidal exchange and dispersion in Boston Harbor. J. Geophys. Res., 97, 15 591-15 606.

Stommel, H., and H. G. Farmer, 1952: On the nature of estuarine circulation, Part I. Woods Hole Oceanographic Institution Tech. Rep. 52-88, WHOI, $131 \mathrm{pp}$.

Takasugi, Y., T. Fujiwara, and T. Higo, 1990: Structure of the strong tidal jet in the Naruto Strait. J. Oceanogr. Soc. Japan, 46, 6983.

Weinstein, M. P., Ed., 1988: Larval Fish and Shellfish Transport through Inlets. American Fisheries Society, 165 pp.

Wheless, G. H., and A. Valle-Levinson, 1996: A modeling study of tidally driven estuarine exchange through a narrow inlet onto a sloping shelf. J. Geophys. Res., 101, 25 675-25 687.

Wolanski, E., E. Drew, K. M. Abel, and J. O'Brien, 1988: Tidal jets, nutrient upwelling and their influence on the productivity of the alga Halimeda in the Ribbon Reefs, Great Barrier Reef. Est. Coast. Shelf Sci., 26, 169-201. 
Copyright $\odot 2003$ EBSCO Publishing 